\title{
When is a Field a Jacobi-Field? A Characterization of States on Tensor Algebras
}

\author{
By
}

\author{
Erwin BRÜNING*
}

\begin{abstract}
In order to analyse the positivity condition for states on the tensoralgebra $E$ over certain (function-)spaces $E$ more efficiently a representation of the components of the states in terms of a set of "independent parameters" is suggested. For this purpose the concept of a Jacobifield is introduced. In the case of a finite dimensional space $E$ every state on $\underline{E}$ is "parametrized" this way. If however the basic space $E$ is infinite dimensional additional domain problems arise related to algebras of unbounded operators which are involved naturally. It is analysed to which extent this "parametrization in terms of Jacobi-fields" also works in the general case, and it is shown that for "many" basic spaces $E$ which occur in applications "most" of the states admit indeed such a "parametrization". This then also means a corresponding decomposition for the associated algebra of unbounded operators into "independent components". Several applications are indicated.
\end{abstract}

\section{Table of Contents}

\&0. Introduction

\&I. Jacobi-Fields and Jacobi-States

1.1. Assumptions and definitions

I.2. On the relation between fields and states

I.3. A first characterization of Jacobi-states

\&II. Density of Jacobi-Statcs

II.1. Spaces with approximation properiy

II.2. Density of Jacobi-states

\&III. Characterization of Jacobi-States

III.1. Decomposition of states

III.2. Functional characterization of Jacobi-states

\& IV. Some Properties of Jacobi-States

IV.1. Convexity and order properties

IV.2. Topological stability

Communicated by H. Araki, July 29, 1985. Revised October 21, 1985.

* Centre de Physique Theorique, ('NRS, I uminy Case 907, 13288 Marscille, Cedex 09 and Universite de Provence. Dept. de Physique Mathematique, Marseille, France.

On leave of absence from the Faculty of Physics, University of Bielefeld. 
\&V. Conclusions

\& VI. Appendix

Acknowledgements, References.

\section{§0. Introduction}

States on the tensoralgebra over a vectorspace $E$ of functions arise naturally in various domains of Physics and Mathematics. With an appropriate choice of the basic function space $E$ they arise in:

i ) general quantum field theory as the sequence of ' $n$-point-functionals' of the field [5],

ii) statistical mechanics as the sequence of correlation functions of a system [20], and

iii) the theory of generalized stochastic processes as the sequence of moments [21].

As our motivation for this subject came from quantum field theory the assumptions about the basic space $E$ are guided by this. But they are general enough not only to cover i) but also a rather general situation for iii). Various parts of the results also apply to ii).

As usual a state $T$ on the tensoralgebra $\underline{E}$ over a space $E$ with involution * is a normalized continuous linear functional on $\underline{E}$ which is positive with respect to the positive cone defined naturally by the product and involution of $\underline{E}$ (for details see Section I). The distinguishing feature of a tensoralgebra $\underline{E}$ is its special grading (compared to topological $*$-algebras in general). This is partly reflected by the fact that a state $T$ on $\underline{E}$ has components $T_{n}$ (the $n$ point-functionals of a field, the correlation functions, or the moments of a generalized stochastic process):

$$
T=\left\{1, T_{1}, T_{2}, \cdots\right\}
$$

The positivity condition implies a complicated chain of inequalities and relations among these components. This fact has prevented any successful analysis of the relations between the components $T_{n}$ of various degree that goes beyond the GNS-construction [2,4]. A "parametrization" of the $T$ in terms of a "set of independent parameters" is the best one can hope for in this context.

In this article we want to isolate a "set of parameters" for states on tensoralgebras and to prove its basic properties. The appropriate notion for this purpose is that of a Jacobi-field (Definition 1.2) and the associated Jacobi- 
states (Definition 1.5).

In order to give a very short (but rough) explanation of the underlying idea we consider the simplest example which is well known, that is we consider the case where the basic space $E$ is one dimensional. Then every state on the tensoralgebra $\underline{E}$ over $E$ can be considered as a sequence $\underline{C}=\left\{1, c_{1}, c_{2}\right.$, $\cdots\}$ of positive type. By the GNS-construction there is a hermitian operator $A$ in a separable Hilbert space with cyclic unit vector $\phi_{0}$ such that

$$
c_{n}=\left\langle\phi_{0}, A^{n} \phi_{0}\right\rangle, n=0,1,2, \cdots
$$

holds. And such an operator $A$ is known [1] to have a representation in terms of a Jacobi-matrix

$$
a=\left(\left(a_{i j}\right)_{i, j=0,1,2, \cdots}\right)
$$

of complex numbers $a_{i j}=\bar{a}_{j i}$; and the numbers

$$
a_{i j}, 0 \leq i \leq j \leq i+1
$$

have to be considered as independent (with some modifications if for some $i a_{i, i+1}=0$ occurs, see Definition 1.3). The components $c_{n}$ of the sequence $\underline{C}$ of positive type now appear as functions of this set of parameters:

$$
c_{n}=f_{n}\left(\left(a_{i j}\right)_{0 \leq i \leq j \leq i+1}\right)=(a \cdots a)_{00} \quad n=1,2, \cdots
$$

(the 00 -component of the $n$-fold matrix product).

Thus for instance,

$$
\begin{aligned}
c_{1} & =a_{00}, c_{2}=\left|a_{00}\right|^{2}+\left|a_{01}\right|^{2}=\left|c_{1}\right|^{2}+\left|a_{01}\right|^{2} \\
c_{3} & =a_{00}{ }^{3}+a_{01} \bar{a}_{01} a_{00}+a_{00} \bar{a}_{01} a_{01}+\bar{a}_{01} a_{11} a_{01} \\
& =c_{2} c_{1}+c_{2}\left(c_{2}-\left|c_{1}\right|^{2}\right)+\left(c_{2}-\left|c_{1}\right|^{2}\right) a_{11} .
\end{aligned}
$$

In the general situation of an infinite dimensional space $E$, various additional complications arise as now an infinite set of operators $\{A(x) \mid x \in E\}$ with cyclic unit vector in a separable Hilbert space occurs which in the general case do not commute and are not bounded. The appropriate concept which generalizes the Jacobi-matrix $a$ of the one-dimensional case is that of a "Jacobifield" and can thus be considered as the corresponding "parametrization" for states on $\underline{E}$.

The concept of a Jacobi-field in quantum field theory is from a physical point of view a rather natural one and has proved to be very useful $[17,19]$. In fact all relativistic quantum fields which have been constructed up to now 
over physical space-time are Jacobi-fields. Our analysis will offer an explanation of this fact.

This article has to be considered as a corrected and extended version of my RIMS preprint $n$. 463 "When is a field a Jacobi-field?" which contains some errors and gaps.

The organization of this article is indicated by the table of contents. Some applications are pointed out at the end of Section V.

\section{§I. Jacobi-Fields and Jacobi-States}

\section{I.1 Assumptions and definitions.}

The basic space $E$ over which we are going to consider the tensoralgebra and states on it is supposed to satisfy the following assumptions:

$E$ is a nuclear barreled space over $\boldsymbol{C}$ with continuous involution $*$ such that all spaces

$$
E_{n}=\tilde{\otimes}_{\pi}^{n} E \quad n=2,3, \cdots
$$

are barreled too.

Here $\tilde{\otimes}_{\pi}^{n} E$ denotes the completed $n$-fold projective tensor product of $E$. In the case of a nuclear Fréchet-space for instance it is known [11,13] that all the spaces $E_{n}$ are nuclear Fréchet-spaces and thus are barreled. Another way of assuring the tensor-product spaces of $E$ with itself to be barreled would be to assume $E$ to be a nuclear $L F$-space and to work with the inductive tensorproduct topology and to proceed along the line of [16]. In any case whenever $(\mathrm{H})$ holds we know all the spaces $E_{n}, n=1,2, \cdots$, to be nuclear barreled spaces with continuous involution (by canonical extension) and therefore the locally convex direct sum

$$
\underline{E}=\bigoplus_{n=0}^{\infty} E_{n}
$$

is a nuclear barreled space with continuous involution $*[11,13]$. Under the product

$$
(\underline{x}, \underline{y}) \rightarrow \underline{x} \circ \underline{y},(\underline{x} \circ \underline{y})_{n}=\sum_{i+j=n} x_{i} \otimes y_{j} \quad \underline{x}, \underline{y} \in \underline{E}
$$

$\underline{E}$ turns out to be a locally convex $*$-algebra with unit

$$
\underline{1}=(1,0,0, \cdots) \text {. }
$$

$E$ is called the (completed) tensoralgebra over $E$.

Definition 1.1. A family $\{A(x) \mid x \in E\}$ of linear operators $A(x): \mathscr{D} \rightarrow \mathscr{D}, \mathscr{D}$ 
a dense subspace of a separable Hilbert space $\mathcal{H}$, is called a field over $E$ iff i ) $x \mapsto A(x)$ is a linear map from $E$ into the algebra $L(\mathscr{D}, \mathscr{D})$ of linear operators $\mathscr{D} \rightarrow \mathscr{D}$.

ii) $x \mapsto A(x) \phi$ is a continuous map from $E$ into $\mathscr{H}$ for every $\phi \in \mathscr{D}$.

iii) $A\left(x^{*}\right) \subseteq(A(x))^{*}$ (=the adjoint of $A(x)$ in $\mathscr{H}$ ) for every $x \in E$.

iv) There is a unit vector $\phi_{0} \in \mathscr{D}$ such that the linear span

$$
\mathscr{D}_{0}=\operatorname{Lin}\left\{A\left(x_{1}\right) \cdots A\left(x_{n}\right) \phi_{0} \mid x_{i} \in E, n=0,1,2 \cdots\right\}
$$

is dense in $\mathscr{H}$, e.g. $\phi_{0}$ is cyclic for $\{A(x) \mid x \in E\}$.

Thus we consider a field $A$ over $E$ to be an element of

$$
\mathcal{L}_{s c c}^{h}(E, L(\mathscr{D}, \mathscr{D}))
$$

where $h$ refers to the hermiticity relation iii), $s c$ to the continuity property ii) and $c$ indicates the existence of a cyclic vector.

If $A$ is a field over $E$ we denote by $\mathscr{H}_{(n)}$ the closed sub-space of $\mathscr{H}$ generated by $\left\{A\left(x_{1}\right) \cdots A\left(x_{j}\right) \phi_{0} \mid x_{i} \in E, j=0,1, \cdots, n\right\}$.

The $n$-field-sector $\mathcal{H}_{n}$ of $A$ is then defined as

$$
\mathscr{H}_{n}=\mathscr{H}_{(n)} \ominus \mathscr{H}_{(n-1)} \quad \text { for } n \geq 1 \text { and } \mathscr{H}_{0}=\mathbb{C}_{\phi_{0}}
$$

e.g. that subspace of $\mathscr{H}_{(n)}$ which is orthogonal to $\mathscr{H}_{(n-1)}$. If $Q_{n}$ denotes the orthogonal projection from $\mathscr{H}$ onto $\mathscr{H}_{n}$ we know $Q_{n} \mathscr{D}$ to be dense in $\mathscr{H}_{n}$, but $Q_{n} \mathscr{D}$ may fail to be in the domain $\mathscr{D}$ of the field $A$.

A Jacobi-field is defined to be a field for which $Q_{n} \mathscr{D}$ always is contained in $\mathscr{D}$. More precisely we have [7].

Definition 1.2. A field $A$ over $E$ with cyclic unit vector $\phi_{0} \in \mathscr{D}$ is called a Jacobi-field (over $E$ ) iff

a) $\mathscr{D}=\bigoplus_{n=0}^{\infty} \mathscr{D}_{n}$ (direct sum), $\mathscr{D}_{0}=\boldsymbol{C} \phi_{0}$,

$\mathscr{D}_{n}$ is dense in the $n$-field sector $\mathscr{H}_{n}, n=1,2, \cdots$

b) There are linear maps $A_{i j}: E \rightarrow L\left(\mathscr{D}_{j}, \mathscr{D}_{i}\right)\left(L\left(\mathscr{D}_{j}, \mathscr{D}_{i}\right)\right.$ the space of linear operators $\mathscr{D}_{j} \rightarrow \mathscr{D}_{i}$ ) for $i, j=0,1,2, \cdots$ such that
i) $A_{i j}=0 \quad$ if $|i-j|>1$
ii) $x \mapsto A_{i j}(x) \phi$ is a continuous map from $E$ into $\mathscr{H}_{\imath}$ for every $\phi \in \mathscr{D}_{j}$.
iii) $A_{i j}\left(x^{*}\right) \subseteq\left(A_{j i}(\mathrm{x})\right)^{*} \quad$ for every $x \in E$ iv) $A(x) \phi=\sum_{i=0}^{n} \sum_{i=j-1}^{j+1} A_{i j}(x) \phi_{j} \quad$ for all $x \in E$ whenever $\phi=\left\{\phi_{0}, \phi_{1}, \cdots, \phi_{n}, 0,0\right.$,
$\cdots\} \in \mathscr{D}$.

Remark 1.1. We mention a simple but important consequence of these 
definitions:

$$
A_{n+1, n}(E) \cdots A_{2,1}(E) A_{1,0}(E) \phi_{0} \text { is total in } \mathscr{H}_{n+1}
$$

for $n=0,1,2 \cdots$.

It turns out to be useful to distinguish special classes of Jacobi-fields. These are defined as follows:

\section{Definition 1.3.}

a) A field $A$ over $E$ is said to be a bounded Jacobi-field iff $A$ is a Jacobi-field such that all the operators $A_{i j}(x), x \in E$, are bounded operators from the Hilbert space $\mathscr{H}_{j}$ into the Hilbert space $\mathscr{H}_{i}$ for all $i, j=0,1,2, \cdots$.

b) A field $A$ over $E$ is called a Jacobi-field of order $N$ iff $A$ is a Jacobi-field such that the state space $\mathcal{H}$ of $A$ is generated by the first $N+1$ field sectors, e.g.

$$
\mathscr{H}=\bigoplus_{n=0}^{N} \mathscr{H}_{n} \quad N \in \boldsymbol{N} \text { minimal. }
$$

If $A$ is a bounded Jacobi-field we can and will assume that the operators $A_{i j}(x)$, $x \in E$, are defined on all of $\mathscr{H}_{j}$ and not only on the dense subspace $\mathscr{D}_{j}$ of $\mathscr{H}_{j}$.

Remark 1.2. The notion of a Jacobi-field has been introduced in axiomatic field theory by Yu. M. Berezanskii and V. D. Koshmanenko in 1969 [19]. Their notion of a Jacobi-field seems to correspond what we have called a bounded Jacobi-field. But already simple examples of relativistic quantum fields such as the Wick-square of a free field [5] show that also Jacobi-fields occur which are not bounded (In this example the diagonal operators $A_{i i}(x)$ are not bounded). From the point of view of classifying fields Jacobi-fields which are not bounded obviously should be considered.

Remark 1.3. Corresponding to the notion of a Jacobi-field of finite order there is the general notion of a field of finite order. A field $A$ over $E$ is called a field of order $N$ iff $N$ is minimal such that the state space $\mathscr{H}$ of $A$ is generated by the first $N+1$ field-sectors [8, 10]:

$$
\mathscr{H}=\bigoplus_{n=0}^{N} \mathscr{H}_{n}=\mathscr{H}_{(N)} \text {. }
$$

Here we will not consider this case further in much details.

\section{I.2. Fields over $E$ and states on the completed tensor algebra over $\underline{E}$.}

If one wants to decide whether a given field $A$ is a Jacobi-field or not this seems to be quite hopeless because of lack of information about the projectors 
$Q_{n}$. Therefore the question whether a given field is "equivalent" (in some useful sense) to a Jacobi-field seems to be more appropriate. In order to explain the following definition of equivalence of fields we have to recall some facts about fields.

The sequence

$$
T^{A}=\left\{1, T_{1}^{A}, T_{2}^{A}, \cdots, T_{n}^{A}, \cdots\right\}
$$

of $n$-point-functionals

$$
T_{n}^{A}\left(x_{1} \otimes \cdots \otimes x_{n}\right)=\left\langle\phi_{0}, A\left(x_{1}\right) \cdots A\left(x_{n}\right) \phi_{0}\right\rangle \quad x_{i} \in E, n \in \mathbb{N}
$$

of a field $A$ over $E$ with cyclic unit vector $\phi_{0}$ uniquely defines a linear functional $T^{A}$ on the tensoralgebra $\mathscr{I}(E)=\bigoplus_{n=0}^{\infty}\left(\otimes_{\pi}^{n} E\right)$ over $E$. This functional turns out to be continuous $[4,6]$ and thus has a unique continuous extension $T^{A}$ to the completion $\underline{E}$ of $\mathscr{I}(E) . \quad T^{A}$ is state of the *-algebra $E$ with unit

$$
T^{A} \in \mathcal{E}(\underline{E})=\left\{T \in \underline{E}^{\prime}=\prod_{n=0}^{\infty} E_{n}^{\prime} \mid T(\underline{1})=1, T\left(\underline{*}^{*} \circ x\right) \geqslant 0 \text { for all } \underline{x \in E}\right\}
$$

( ${ }^{\prime}$ indicates the topological dual).

By the GNS-construction [2-5] every state $T \in \mathcal{E}(\underline{E})$ on $\underline{E}$ gives rise to a strongly continuous hermitian representation $\underline{A}_{T}$ of the $*$-algebra $\underline{E}$ by linear operators on a dense invariant subspace $\mathscr{D}_{T}$ of a separable Hilbert space with a cyclic unit vector $\phi_{0}=\phi_{0}^{T} \in \mathscr{D}_{T}$ such that

$$
T(\underline{x})=\left\langle\phi_{0}, \underline{A}_{T}(\underline{x}) \phi_{0}\right\rangle \quad \text { for all } \underline{x} \in \underline{E}
$$

holds. And such a representation $\underline{A}_{T}$ defines by restriction to $E$ a field $A_{T}$ over $E: A_{T}:=A_{T} \uparrow E$. If $T=T^{A}$ is the sequence of $n$-point-functionals of a field over $E$ it is quite natural to consider the fields $A$ and $A_{T^{A}}$ "essentially as the same". Therefore we propose

Definition 1.4. Two fields (over $E$ ) $A$ and $B$ are said to be equivalent iff their sequences $T^{A}$ resp. $T^{B}$ of $n$-point-functionals agree (on $\mathcal{L}(E)$ or $\left.\underline{E}\right): T^{A}=T^{B}$.

It is instructive to note which possibilities are left for equivalent fields. Besides the obvious one of unitary equivalence there are in general quite a lot of other possibilities. These possibilities are most conviently described in terms of the associated representation $\underline{A}_{T}$ of $\underline{E}$ for a given $T \in \mathcal{E}(\underline{E})[3,7]$. Any strongly continuous hermitian representation $\underline{A}$ of $\underline{E}$ in $\mathscr{H}_{T}$ such that:

i) $\underline{A}_{T} \subseteq \underline{A} \subseteq \underline{\hat{A}}_{T}$ (there exists always a maximal hermitian extension $\underline{\hat{A}}_{T}$ of $\underline{A}_{T}$ which is strongly continuous) or 
ii) $\underline{A} \subseteq \underline{A}_{T}$ but with $\underline{A}(\underline{E}) \phi_{0}$ dense in $\mathscr{A}_{T}$

yields according to equation (1.9) the same state $T$ on $\underline{E}$ and thus all fields $A=\underline{A} \uparrow E$ where $\underline{A}$ satisfies i) or ii) are equivalent to the field $A_{T}=\underline{A}_{T} \uparrow E$ which is given by GNS-construction.

Note that the corresponding phenomenon occurs if a single hermitian operator with a cyclic unit vector is to be represented by a Jacobi-matrix (of numbers).

The following definition of a Jacobi-state takes care of this nonuniqueness of the associated fields.

Definition 1.5. A state $T \in \mathcal{E}(E)$ is called a Jacobi-state iff the associated field $A_{T}$ (by GNS-construction) is equivalent to a Jacobi-field. The set of all Jacobi-states on $\underline{E}$ is denoted by $\mathcal{E}_{J}(\underline{E})$. The set of all states $T$ on $\underline{E}$ such that $A_{T}$ is equivalent to a bounded Jacobi-field is denoted by $\mathcal{E}_{J b}(\underline{E})$.

According to this definition we have

$$
\mathcal{E}_{J b}(\underline{E}) \subsetneq \mathcal{E}_{J}(\underline{E}) \subseteq \mathcal{E}(\underline{E})
$$

and within each class of fields one could distinguish the class of states of finite order and that class of states which are not of finite order thus obtaining a first classification of states on the tensoralgebra $E$. A characterization of those states $T$ on $\underline{E}$ such that the associated GNS-field $A$ is (equivalent to) a field of finite order is given in Theorem 2 of [10]. This characterization is entirely in terms of the state itself not referring to the associated field. Similarly in Section III a characterization for a state on $\underline{E}$ to be a Jacobi-state will be given using only properties of the state.

\section{I.3. A first characterization of Jacobi-states.}

By definition the projection operators $Q_{n}$ map the domain $\mathscr{D}$ of a Jacobifield into $\mathscr{D}$. By the following proposition we learn that this property is up to equivalence characteristic for Jacobi-fields.

Proposition 1.1. If $A$ is a field over $E$ with domain $\mathscr{D}, \overline{\mathscr{D}}=\mathscr{H}$, and if the projections $Q_{n}$ on the $n$-field sectors $\mathcal{H}_{n}$ of $A$ map the domain into itself, $Q_{n} \mathscr{D}$ $\subset \mathscr{D}, n=1,2, \cdots$ then $A$ is equivalent to a Jacobi-field.

Proof. If $\phi_{0}$ is the cyclic unit vector of $A$, define $\mathscr{D}_{0}=C \phi_{0}$ and $\mathscr{D}_{n}=Q_{n} \mathscr{D}$. Then $\mathscr{D}_{n}$ is dense in $\mathscr{H}_{n}, n=1,2, \cdots$ and the direct sum $\hat{\mathscr{D}}=\bigoplus_{n=0}^{\infty} \mathscr{D}_{n}$ is dense in $\mathscr{H}$ as $\mathscr{D}$ is dense in $\mathscr{H}=\bigoplus_{n=0}^{\infty} \mathscr{H}_{n}$. By assumption all the operators are well 
defined. As $A$ is strongly continuous on $\mathscr{D}$ (property ii) of Definition 1.1) we get that $A_{i j}$ is strongly continuous on $\mathscr{D}_{j}$ (property b ii) of Definition 1.2). Hermiticity of $A$, e.g. property iii) of Definition 1.1, immediately implies

$$
\left(A_{i j}(x)\right)^{*}=A_{j i}\left(x^{*}\right) \quad \text { for all } x \in E \text { and } i, j=0,1,2, \cdots \text {. }
$$

By definition of the subspaces $\mathscr{H}_{(n)}$ we have

$$
A(x) \mathscr{D} \cap \mathscr{H}_{(n)} \subseteq \mathscr{D} \cap \mathscr{H}_{(n+1)}
$$

and therefore for all $\phi \in \mathscr{D} \cap \mathcal{H}_{(n)}$, all $x \in E$, and all $i \geqslant n+2$

$$
Q_{i} A(x) \phi=0
$$

and thus $A_{i j}=0$ for $i-j \geqslant 2$. Because of the hermiticity relation for the $A_{i j}$ we get $A_{i j}=0$ whenever $|i-j| \geqslant 2$.

If $\hat{A}(x)=\left(A_{i j}(x), i, j=0,1,2, \cdots\right)$ denotes this matrix of operators we get $\hat{A} \uparrow \hat{\mathscr{D}}=A$ : for any $\phi=\left\{\phi_{0}, \phi_{1}, \cdots, \phi_{N}, O, \cdots\right\} \in \hat{\mathscr{D}} \subset \mathscr{D}, \phi_{n} \in \mathscr{D}_{n} \subset \mathscr{D}$ we have

$$
A(x) \phi=\sum_{j=0}^{N} A(x) \phi_{j}=\sum_{i=0}^{\infty} \sum_{j=0}^{N} Q_{i} A(. x) Q_{j} \phi_{j}=\sum_{|i-j| \leqslant 1} \sum_{j=0}^{N} A_{i j}(x) \phi_{j}=\hat{A}(. x) \phi .
$$

This implies that $\hat{A}$ is a Jacobi-field over $E$ with domain $\hat{\mathscr{D}}$ and cyclic vector $\phi_{0}$ such that $T^{\hat{A}}=T^{A}$ holds. Therefore $A$ is equivalent to the Jacobi-field $\hat{A}$.

If $A$ is a field over $E$ with cyclic vector $\phi_{0}^{A}$, domain $\mathscr{D}$, and statespace $\mathscr{H}_{A}=\overline{\mathscr{D}}$ it defines by canonical extension a hermitian representation $\underline{A}$ of the (incomplete tensoralgebra $\mathscr{L}(E)$ over $E$ ) on $\mathscr{D}$. This representation has as minimal dense invariant domain just

$$
\mathscr{D}_{0}^{A}=\underline{A}(\mathscr{I}(E)) \phi_{0}^{A} \subset \mathscr{D} .
$$

If $B$ is an equivalent field over $E$ with cyclic unit vector $\phi_{0}^{B}$ and statespace $\mathscr{H}_{B}$ an isometric map $U$ is well defined from $\mathscr{D}_{0}^{A}$ onto the minimal dense invariant domain $\mathscr{D}_{0}^{B}$ for the representation $\underline{B}$ of $\mathscr{I}(E)$ associated with $B$ by

$$
U \underline{A}(\underline{x}) \phi_{0}^{A}=\underline{B}(\underline{x}) \phi_{0}^{B} \quad \text { for all } \underline{x} \in \mathscr{I}(E) \text {. }
$$

By continuous extension a unitary map $U: \mathscr{H}_{A} \rightarrow \mathscr{H}_{B}$ is obtained such that

$$
U \mathcal{H}_{(n)}^{A}=\mathscr{H}_{(n)}^{B} \quad \text { for } n=0,1,2, \cdots .
$$

It follows that $U$ maps the fieldsector $\mathcal{H}_{n}^{A}$ of $A$ onto the field-sector $\mathcal{H}_{n}^{B}$ of $B$,

$$
U \mathscr{H}_{n}^{A}=\mathscr{H}_{n}^{B} \quad n=0,1,2, \cdots .
$$

Therefore up to a unitary transformation of the statespace equivalent fields have the same field sectors. 
Together with (1.10) this observation implies the following corollary to Proposition 1.1.

Corollary 1.2. Let $T$ be a state on $\underline{E}$ and $A_{T}$ its associated GNS-field on its minimal invariant dense domain $\mathscr{D}_{0}^{T} \subset \mathcal{A}_{T}$. Then $T$ is a Jacobi-state iff $A_{T}$ has an extension $A \in \mathcal{L}_{s c c}^{h}(E, L(\mathscr{D}, \mathscr{D}))$ in $\mathscr{H}_{T}$ (e.g. $A$ is a field such that $\mathscr{D}_{0}^{T} \subseteq$ $\mathscr{D} \subseteq \mathscr{H}_{T}$ and $\left.A(x) \uparrow \mathscr{D}_{0}^{T}=A_{T}(x), x \in E\right)$ such that for the domain $\mathscr{D}$ of $A$ and the projections $Q_{n}$ onto the field-sectors of $A$ the relations $Q_{n} \mathscr{D} \subseteq \mathscr{D}$ hold for $n=$ $0,1,2, \cdots$.

Proof. If $T$ is a Jacobi-state it can be regarded as the sequence of $n$ point-functionals of a Jacobi-field $A$. This Jacobi-field $A$ can be supposed to be realized in the GNS-Hilbertspace for $T$ and then satisfies the conditions of the corollary. Conversely if these conditions are satisfied for the extension $A$ of $A_{T}$ it follows $T=T^{A}$. By Proposition $1.1 A_{T}$ is equivalent to a Jacobifield. Therefore $T$ is a Jacobi-state.

\section{§III. Density of Jacobi-States}

\section{II.1. Spaces with approximation property.}

In order to prove that the set $\mathcal{E}_{J}(\underline{E})$ of all Jacobi-states is dense in the set of all states $\mathcal{E}(\underline{E})$ on $\underline{E}$ a certain approximation property of the basic space $E$ is used. The appendix contains a short discussion of this property and shows in particular that many important spaces $E$ which actually occur in applications have this approximation property.

\section{Definition 2.1.}

a) A locally convex topological vector space $E$ is said to have property (a) iff there is a net $\left(\pi_{\gamma}\right)_{\gamma \in \Gamma}$ of continuous linear maps $\pi_{\gamma}: E \rightarrow E$ such that

i) $\operatorname{dim} \operatorname{Ran} \pi_{\gamma}<\infty \quad$ for all $\gamma \in \Gamma$

ii) $\lim _{\gamma \in \Gamma} \pi_{y}(x)=x$ in $E \quad$ for all $x \in E$

iii) $\left\{\pi_{\gamma}(x) \mid \gamma \in \Gamma\right\}$ is bounded in $E$ for every $x \in E$.

b) A locally convex topological vector space $E$ is said to have property (a) with respect to sequences or property (as) iff there is a sequence $\left\{\pi_{n}\right\}_{n \in N}$ of continuous linear maps $\pi_{n}: E \rightarrow E$ such that

i) $\operatorname{dim} \operatorname{Ran} \pi_{n}<\infty \quad$ for all $n \in \mathbb{N}$

ii) $\lim _{n \rightarrow \infty} \pi_{n}(x)=x$ in $E \quad$ for all $x \in E$.

Suppose now that the space $E$ satisfies $(\mathrm{H})$ and has property (a). As $E$ is supposed to be barreled we get immediately that $\left\{\pi_{\gamma} \mid r \in \Gamma\right\}$ is an equi- 
continuous subset of $\mathcal{L}(E, E)$ (Theorem III 4.2 of [11]). We can and will assume that $\pi_{\gamma}^{*}=\pi_{\gamma}$ holds for all $\gamma \in \Gamma$. Otherwise we would take $\hat{\pi}_{\gamma}=\left(\pi_{\gamma}+\right.$ $\left.\pi_{\gamma}^{*}\right) / 2$ where the definition $\pi_{\gamma}^{*}(x):=\left(\pi_{\gamma}\left(x^{*}\right)\right)^{*}$ is used. $\pi_{\gamma}^{*}$ is again a continuous linear map with finite dimensional range $\operatorname{Ran} \pi_{\gamma}^{*}=\left(\operatorname{Ran} \pi_{\gamma}\right)^{*}$ and $\lim \pi_{\gamma}^{*}(x)$ $=\left(\lim \pi_{\gamma}\left(x^{*}\right)\right)^{*}=\left(x^{*}\right)^{*}=x$ for all $x \in E$. Thus $\hat{\pi}_{\gamma}$ has the same properties and satisfies $\hat{\pi}_{\gamma}^{*}=\hat{\pi}_{\boldsymbol{\gamma}}$.

For every $r \in \Gamma$ we define $\underline{\pi}_{\gamma}: \underline{E} \rightarrow \underline{E}$ by

$$
\underline{\pi}_{\gamma}:=\bigoplus_{n=0}^{\infty} \pi_{\gamma}^{\otimes n} \quad \pi_{\gamma}^{\otimes 0}=1 .
$$

These maps are homomorphisms of the $*$-algebra $\underline{E}$ with unit, e.g. for all $\underline{x}$, $\underline{y} \in \underline{E}$ and all $r \in \Gamma$ we have

$$
\underline{\pi}_{\gamma}(\underline{1})=\underline{1}, \quad\left(\underline{\pi}_{\gamma}(\underline{x})\right)^{*}=\underline{\pi}_{\gamma}\left(\underline{x}^{*}\right), \quad \underline{\pi}_{\gamma}(\underline{x} \cdot \underline{y})=\pi_{\gamma}(\underline{x}) \underline{\pi}_{\gamma}(\underline{y}) .
$$

Furthermore we will show

$$
\begin{gathered}
\left\{\underline{\pi}_{\gamma} \mid \gamma \in \Gamma\right\} \quad \text { is equicontinuous in } \mathcal{L}(\underline{E}, \underline{E}) \\
\lim _{\gamma \in \Gamma} \underline{\pi}_{\gamma}(\underline{x})=\underline{x} \text { in } \underline{E} \text { for all } \underline{x} \in \underline{E} . \\
\operatorname{Ran} \underline{\pi}_{\gamma}=\mathscr{I}\left(\operatorname{Ran} \pi_{\gamma}\right) \text { for all } \gamma \in \Gamma
\end{gathered}
$$

If $q_{(n)}$ is a continuous seminorm on $E_{n}$ there are continuous seminorms $q_{1} \cdots q_{n}$ on $E$ such that $q_{(n)} \leq q_{1} \otimes_{\pi} \cdots \otimes_{\pi} q_{n}$.

As $\left\{\pi_{\gamma} \mid \gamma \in \Gamma\right\}$ is equicontinuous in $\mathcal{L}(E, E)$ there are continuous seminorms $p_{1}, \cdots, p_{n}$ on $E$ such that $q_{j}\left(\pi_{\gamma}(x)\right) \leq p_{j}(x)$ holds for all $r \in \Gamma, x \in E$, and $j=1$, $\cdots, n$.

It follows

$$
q_{(n)}\left(\pi_{\gamma}^{\otimes n}(x)\right) \leq\left(p_{1} \otimes_{\pi} \cdots \otimes_{\pi} p_{n}\right)(x) \text { for all } \gamma \in \Gamma \text { and } x \in E_{n} .
$$

Therefore $\left\{\pi_{\tau}^{\otimes n} \mid \gamma \in \Gamma\right\}$ is equicontinuous in $\mathcal{L}\left(E_{n}, E_{n}\right)$ for $n=1,2, \cdots$ and thus $\left\{\underline{\pi}_{\gamma} \mid \gamma \in \Gamma\right\}$ is equicontinuous in $\mathcal{L}(\underline{E}, \underline{E})$. For any $x=x_{1} \otimes \cdots \otimes x_{n} \in \otimes^{n} E$ we have

$$
\begin{aligned}
x-\pi_{\gamma}^{\otimes n}(x)= & x_{1} \otimes \cdots \otimes x_{n-1} \otimes\left(x_{n}-\pi_{\gamma}\left(x_{n}\right)\right)+\left(x_{1} \otimes \cdots \otimes x_{n-1}\right. \\
& \left.-\pi_{\gamma}^{\otimes(n-1)}\left(x_{1} \otimes \cdots \otimes x_{n-1}\right)\right) \otimes \pi_{\gamma}\left(x_{n}\right)
\end{aligned}
$$

As $\left\{\pi_{\gamma}\left(x_{n}\right) \mid \gamma \in \Gamma\right\}$ is bounded in $E$ we get by induction on $n$

$$
x=\lim _{\gamma \in \Gamma} \pi_{\gamma}^{\otimes n}(x) \text { for all } x \in \otimes^{n} E
$$

and therefore $\quad \underline{x}=\lim _{\gamma \in \Gamma} \underline{\pi}_{\gamma}(\underline{x})$ for all $\underline{x} \in \mathscr{L}(E)$.

This implies (2.4) because $\mathscr{I}(E)$ is dense in $\underline{E}$ and $\left\{\underline{\pi}_{\gamma} \mid \gamma \in \Gamma\right\}$ is equicontinuous 
The following relations for $n=1,2, \cdots$ and $r \in \Gamma$ are obvious:

$$
\pi_{\gamma}^{\otimes n}\left(\bigotimes^{n} E\right)=\bigotimes^{n} \operatorname{Ran} \pi_{\gamma}
$$

$\otimes^{n} \operatorname{Ran} \pi_{\gamma}$, being finite dimensional, is a closed subspace of $E_{n} ;$ thus $\operatorname{Ran} \pi_{\gamma}^{\otimes n}$ $=\pi_{\gamma}^{\otimes n}\left(E_{n}\right)=\otimes^{n} \operatorname{Ran} \pi_{\gamma}$ and therefore

$$
\operatorname{Ran} \underline{\pi}_{\gamma}=\bigotimes_{n=0}^{\infty} \operatorname{Ran} \pi_{\gamma}^{\otimes n}=\mathscr{I}\left(\operatorname{Ran} \pi_{\gamma}\right)
$$

e.g. (2.5)

\section{II.2. Density of Jacobi-states.}

If the underlying space $E$ satisfies $(\mathrm{H})$ and has property (a) there is a net $\left(\pi_{\gamma}\right)_{\gamma \in \Gamma}$ of continuous linear maps $E \rightarrow E$ according to definition (2.1). Then the associated continuous linear maps $\underline{\pi}_{\gamma}: \underline{E} \rightarrow \underline{E}$ defined by eq (2.1) have properties (2.2)-(2.5). Thus if $T$ is a state on $\underline{E}$ we define for $r \in \Gamma$

$$
T^{\gamma}=T \circ \underline{\pi}_{\gamma}
$$

and get immediately by (2.2) and (2.3) that $\left\{T^{\gamma} \mid \gamma \in \Gamma\right\}$ is an equicontinuous net of states on $\underline{E}$. By (2.4) it is clear that

$$
\lim _{\gamma \in \Gamma} T^{\gamma}(\underline{x})=T(\underline{x})
$$

holds for every $\underline{x} \in \underline{E}$. The Banach-Steinhaus Theorem (\$39.5.1 of [13]) implies that the above convergence is uniform on every precompact subset of $\underline{E}$. Thus if we could show the $T^{\gamma}$ to be Jacobi-states we had specified a notion of convergence with respect to which the set of Jacobi-states is dense in $\varepsilon(E)$. In order to do so we recall first the following definition [6].

Defimition 2.2. A state $T \in \mathcal{E}(\underline{E})$ is said to be finite dimensional iff the range of the associated GNS-field $A_{T}$ is finite dimensional, e.g. if $A_{T}(E)$ is a finite dimensional space of operators.

Remark 2.1. In [6] this class of states is characterized by the equivalent condition that the factorspace $E / I(T) \cap E$ is finite dimensional. Here $I(T)$ denotes the maximal two-sided ideal in $\underline{E}$ which is contained in the leftkernel $L(T)=\left\{\underline{x} \in \underline{E} \mid T\left(\underline{x}^{*} \cdot \underline{x}\right)=0\right\}$ of the state $T$. If $\underline{A}_{T}$ denotes the GNS-representation of $\underline{E}$ associated with $T$ one has $I(T)=\operatorname{Ker} \underline{A}_{T}$.

Proposition 2.1. Every finite dimensional state on $\underline{E}$ is a bounded Jacobistate, e.g.

$$
\varepsilon_{f}(E) \subset \varepsilon_{J b}(\underline{E})
$$


if $\mathcal{E}_{f}(\underline{E})$ denotes the set of all finite dimensional states on $\underline{E}$.

Proof. For a finite dimensional state $T$ on $\underline{E}$, we know that the subspaces

$$
\underline{A}_{T}\left(\underline{E}_{(n)}\right) \phi_{0}^{T}, \underline{E}_{(n)}=\left\{\underline{x} \in \mathscr{I}(E) \mid x_{j}=0 \text { for all } j>n\right\}
$$

in the GNS-representation of $T$ are finite dimensional. It follows

$$
\mathscr{H}_{(n)}=\left[\underline{A}_{T}\left(\dot{E}_{(n)}\right) \phi_{0}^{T}\right]=\underline{A}_{T}\left(\dot{E}_{(n)}\right) \phi_{0}^{T} \subset \mathscr{D}_{T}
$$

and $\operatorname{dim} \mathscr{H}_{(n)}<\infty$. Therefore the $n$-field-sectors

$$
\mathscr{H}_{n}=\mathscr{H}_{(n)} \ominus \mathscr{H}_{(n-1)}
$$

in this case are finite dimensional subspaces of the domain $\mathscr{D}_{T}$ of the GNSrepresentation and this implies $O_{n} \mathscr{D}_{T} \subseteq \mathscr{D}_{T}$ for all $n=0,1,2, \cdots$. By Proposition $1.1 A_{T}$ is equivalent to a Jacobi-field which is obviously bounded as the fieldsectors are finite dimensional; thus $T$ is a bounded Jacobi-state.

Together with the remarks at the beginning of this subsection this proposition immediately implies the density of Jacobi-states.

Theorem 2.2. Suppose $E$ satisfies $(\mathrm{H})$ and has property (a). Then for cvery state $T \in \mathcal{E}(\underline{E})$ on $\underline{E}$ there is a net $\left(T^{\gamma}\right)_{\gamma \in \Gamma}$ of finite dimensional states $T^{\gamma} \in \mathcal{E}_{f}(\underline{E})$ and a continuous hilbertian seminorm $\underline{q}$ on $\underline{E}$ such that

i) $\left|T^{\gamma}\right| \leq q$ for all $\gamma \in \Gamma$

ii) $T(\underline{x})=\lim _{\gamma \in \Gamma} T^{\gamma}(\underline{x})$ uniformly in $\underline{x} \in K$ for every precompact subset $K \subset \underline{E}$.

Proof. a) If $T \in \mathcal{E}(E)$ is given define $T^{\gamma}$ according to (2.6). Then the net $\left\{T^{\gamma} \mid \gamma \in \Gamma\right\}$ is equicontinuous. Thus there is a continuous seminorm $\underline{q}$ on $E$ such that i) holds. As $\underline{E}$ is nuclear this seminorm can be chosen to be hilbertian. Relation (2.7) and the remark following it prove ii).

b) Let us denote by $\underline{A}=\left(\underline{A}, \phi_{0}, \mathscr{D}=\underline{A}(\underline{E}) \phi_{0}, \mathscr{H}\right)$ the GNS-representation of $T$ where the specification of the cyclic unit vector $\phi_{0}$, the domain $\mathscr{D}$ and the state space $\mathscr{H}$ is included and by $\underline{A}^{\gamma}=\left(\underline{A}^{\gamma}, \phi_{0}^{\gamma}, \mathscr{D}^{\gamma}=\underline{A}^{\gamma}(\underline{E}) \phi_{0}^{\gamma}, \mathcal{H}^{\gamma}\right)$ that of $T^{\gamma}$. Then we have for all $\underline{x} \in \underline{E}$

$$
\left(\phi_{0}^{\gamma}, \underline{A}^{\gamma}(\underline{x}) \phi_{0}^{\gamma}\right)_{\mathcal{G l}_{l}}{ }^{\prime}=T^{\gamma}(\underline{x})=T\left(\underline{\pi}_{\gamma}(\underline{x})\right)=\left(\phi_{0}, \underline{A}\left(\underline{\pi}_{\gamma}(\underline{x})\right) \phi_{0}\right)_{\mathscr{H}} .
$$

Therefore $A^{\gamma}$ is unitarily equivalent to the following representation of $\underline{E}$ in $\mathscr{H}$ :

$$
\hat{A}^{\gamma}=\left(\underline{A} \circ \pi_{\gamma}, \phi_{0}, \underline{\Lambda}\left(\mathcal{I}\left(\operatorname{Ran} \pi_{\gamma}\right)\right) \phi_{0},\left[\underline{A}\left(\mathcal{I}\left(\operatorname{Ran} \pi_{\gamma}\right)\right) \phi_{0}\right]\right) .
$$

Here equation (2.5) is used and [M] denotes the closed subspace of $\mathscr{H}$ generated 
by a set $M \subseteq \mathscr{H}$. In this representation we have

$$
\hat{A}^{\gamma}(E)=A\left(\pi_{\gamma}(E)\right)
$$

and therefore $\operatorname{dim} \hat{A}^{\gamma}(E) \leq \operatorname{dim} \operatorname{Ran} \pi_{\gamma}<\infty$, that is $T^{\gamma}$ is a finite dimensional state on $\underline{E}$ for each $r \in \Gamma$ and this proves Th. 2.2.

Using some general results about weak topologies one can give a stronger version of Theorem 2.2. As a nuclear space is separable with respect to each of its continuous seminorms Th. III 4.7 of [11] implies:

Lemma 2.3. Let $F$ be nuclear space with topological dual $F^{\prime}$ and $H \subset F^{\prime}$ be an equicontinuous subset. Then the weak topology of $F^{\prime}$ restricted to $H$ is metrizable, e.g. $\sigma\left(F^{\prime}, F\right) \uparrow H$ is metrizable.

In the situation of Theorem 2.2 every state $T$ on $E$ is the weak limit of an equicontinuous net $\left(T^{\gamma}\right)_{\gamma \in \Gamma}$ of finite dimensional states. As $E$ is nuclear we can apply Lemma 2.3 to $H=\left\{T^{\gamma} \mid \gamma \in \Gamma\right\} \cup\{T\}$ and obtain that $\sigma\left(E^{\prime}, E\right)$ $\uparrow H$ is metrizable. Therefore we can select a subsequence $T^{j}=T^{\gamma(\jmath)}, j \in N$, converging weakly to $T$.

Corollary 2.4. Suppose $E$ satisfies $(\mathrm{H})$ and has property (a). Then every state $T$ on $\underline{E}$ is the weak limit of a sequence $\left(T^{j}\right), j \in \mathbb{N}$ of finite dimensional states, e.g. $\mathcal{E}(\underline{E})$ is the weak sequential closure of $\mathcal{E}_{f}(\underline{E})$ and thus in particular of $\mathcal{E}_{J b}(\underline{E})$ or $\varepsilon_{J}(\underline{E})$.

Remark 2.2. For $E=\mathcal{S}\left(\boldsymbol{R}^{n}\right)$, the Schwartz space of rapidly decreasing $C^{\infty}$-functions on $\mathbb{R}^{n}$, H.J. Borchers proved in [6] that the set of finite dimensional states is weakly dense in the set of all states on $E$. As this space is known to be a nuclear Fréchet space it satisfies our hypothesis (compare appendix). Thus this result is covered by Corollary 2.4 which provides however important additional information.

\section{§III. Characterization of Jacobi-States}

III.1. Decomposition of states.

In the introduction it was claimed that a Jacobi-field provides a parametrization of the Jacobi-states in the following sense: every component $T_{n} \in E_{n}^{\prime}$ of a Jacobi-state $T \in \mathcal{E}_{j}(\underline{E})$ appears as a function

$$
T_{n}=T_{n}\left(\left(A_{i j}\right)_{i, j=0,1,2, \ldots)}\right.
$$

of the independent parameters $A_{i j}, 0 \leq i \leq j \leq i+1, i+1 \leq[n / 2]$. 
But as we have seen in Section 1.3 there is some freedom with respect to extensions or restrictions of the associated Jacobi-field $A=\left(A_{i j} \mid i, j=0,1\right.$, 2 ...) such that (3.1) holds. Therefore the goal of this Section III is to eliminate this freedom in the parametrization (3.1) which is due to domain problems for unbounded operators involved, and to present the corresponding characterization entirely in terms of properly chosen functionals. This is achieved by introducing the notion of a "Jacobi-decomposition" of a state in such a way that

i) the sequence of $n$-point-functionals of a Jacobi-field has this decomposition property

ii) if a state allows a Jacobi-decomposition then it is a Jacobi-state (in the sense of Definition 1.5).

Obviously i) has to be more or less the definition of a Jacobi-decomposition while ii) needs a proof.

By its very definition a Jacobi-field $A$ associates to every $x \in E$ an infinity of linear operators $\left\{A_{i j}(x)|i, j=0,1,2, \cdots| i-j \mid, \leq 1\right\}$ in some Hilbert space.

In principle this fact can be described by saying that $A$ arises by GNS-construction from a state on a much bigger algebra than $\underline{E}$ in which however $\underline{E}$ is embedded. (Formally $E$ has to be replaced by

$$
F=\prod_{i, j=0}^{\infty} F_{i, j} \quad \text { (only for }|i-j| \leq 1 \text { ) }
$$

and the big algebra in question is a certain subalgebra of the tensoralgebra over $F$ with appropriate definitions of the product and the involution).

As in this approach one cannot restrict to direct sums of finite tensor products various additional topological problems arise. This would imply that this approach though in some sense being more elegant is actually more complicated than necessary.

If one restricts to reconstruct only the structure of the underlying Hilbert space and not this big algebra of unbounded operators the situation is much simpler and more direct as it now suffices to consider only direct sums of finite tensorproducts of $E$ which is much easier. But instead of states on an algebra one now only has certain non-negative sesquilinear-forms on a certain space $\underline{E}$. This nevertheless yields a complete description of those states on $\underline{E}$ arising from a Jacobi-field and allows to discuss various important properties of the set of all Jacobi-states. We begin by introducing some notations. For $n \in \mathbb{N}$ we denote:

$$
\mathscr{g}(n)=\left\{I=\left(i_{1}, \cdots, i_{n}\right)\left|i_{j} \in \mathbb{N},\right| i_{j+1}-i_{j} \mid \leq 1, j=1, \cdots, n-1, i_{n} \leq 1\right\}
$$




$$
g_{k}(n)=\left\{I \in g(n) \mid i_{1}=k\right\} .
$$

Obviously $g_{k}(n)=\emptyset$ if $k>n$ and $g_{n}(n)$ contains exactly one element $I=I(n)$ $=(n, n-1, \cdots, 2,1)$.

For $I=\left(i_{1}, \cdots, i_{n}\right) \in \mathcal{g}(n)$ we define $I^{*}=\left(i_{n}, \cdots, i_{2}, i_{1}\right)$.

For $J \in g_{k}(n)$ and $I^{*} \in \mathscr{g}_{k}(m)$ we denote

$$
I \dot{\times} J=\left(i_{1}, \cdots, i_{m-1}, i_{m}=k=j_{1}, j_{2}, \cdots, j_{n}\right) \in \mathscr{g}(n+m-1) .
$$

Furthermore we introduce

$$
[k, \measuredangle]^{r+1}=\left\{\left(i_{1}, \cdots, i_{r+1}\right)\left|i_{j} \in N,\right| i_{j+1}-i_{j} \mid \leqslant 1, j=1, \cdots, r . i_{1}=k, i_{r+1}=\ell\right\}
$$

and obtain for $H \in[k, \iota]^{r+1}$ and $I \in g_{\iota}(n)$

$$
H \dot{\times} I \in \mathscr{g}_{k}(n+r) .
$$

With these notations we can describe Jacobi-fields and the associated sequence of $n$-point-functionals in more details. Assume a Jacobi-field $A$ $=\left(\left(A_{i j}\right) i, j=0,1,2, \cdots\right)$ to be given according to Definition 1.2 with cyclic unit vector $\phi_{0}$ in the Hilbertspace $\mathscr{H}$. In order to describe the minimal dense invariant domain for $A$ and its action on it we introduce some abbreviations. For $I \in g_{k}(n)$ and $x_{1}, \cdots, x_{n} \in E$

$$
\psi_{I}\left(x_{1} \otimes \cdots \otimes x_{n}\right)=A_{i_{1} i_{2}}\left(x_{1}\right) A_{i_{2} i_{3}}\left(x_{2}\right) \cdots A_{i_{n} 0}\left(x_{n}\right) \phi_{0}
$$

and for $H \in[k, \ell]^{r+1}$ and $x_{1}, \cdots, x_{r} \in E$

$$
A_{H}\left(x_{1} \otimes \cdots \otimes x_{r}\right)=A_{i_{1} t_{2}}\left(x_{1}\right) A_{i_{2} i_{3}}\left(x_{2}\right) \cdots A_{i_{r i r+1}}\left(x_{r}\right) .
$$

It follows

$$
A_{H}(z) \psi_{I}(x)=\psi_{H} \dot{\times}_{I}(z \otimes x)
$$

for all $I \in \mathcal{g}_{\ell}(n), H \in[k, \iota]^{r+1}, x \in E^{\otimes n}, z \in E^{\otimes r}$.

In terms of its "components" the action of the Jacobi-field $A$ is now given by

$$
A\left(x_{1}\right) \cdots A\left(x_{n}\right) \phi_{0}=\bigoplus_{k=0}^{n} \sum_{I \in \mathcal{g}_{k}(n)} \psi_{I}\left(x_{1} \otimes \cdots \otimes x_{n}\right)
$$

for all $x_{i} \in E, n=1,2, \cdots$. The orthogonal decomposition in (3.5) arises because of the orthogonality of the field-sectors $\mathscr{H}_{k}$. Note that by definition (3.2) we have for all $n \geqslant k$ all $x \in E^{\otimes n}$ and all $I \in \mathscr{g}_{k}(n)$

$$
\psi_{I}(x) \in \mathscr{H}_{k} .
$$

If we define now for $k=1,2, \cdots$

$$
\mathscr{D}^{k}=\operatorname{lin} \bigcup_{n \geqslant k} \bigcup_{I \in \mathscr{g}_{k}(n)} \psi_{1}(E(I))
$$


where $E(I)=E^{\otimes n} \quad$ for $I \in g_{k}(n)$ and

$$
\hat{\mathscr{D}}=\bigoplus_{k=0}^{\infty} \mathscr{D}^{k} \quad \mathscr{D}^{0}=\boldsymbol{C} \phi_{0} \quad \text { (direct sum) }
$$

we obtain the minimal dense invariant domain $\hat{\mathscr{D}}$ for the Jacobi-field $A$. By Remark 1.1 we know that

$$
\mathscr{D}_{k}=\psi_{I(k)}\left(E^{\otimes k}\right) \quad k=1,2, \cdots
$$

is already dense in the $k$-field sector $\mathscr{H}_{k}$.

Relation (3.5) implies the following decomposition of the $n$-point-functionals

with

$$
T_{n}\left(x_{1} \otimes \cdots \otimes x_{n}\right)=\sum_{I \in \mathcal{G}_{0}(n)} T_{I}\left(x_{1} \otimes \cdots \otimes x_{n}\right)
$$

$$
\begin{aligned}
T_{I}\left(x_{1} \otimes \cdots \otimes x_{n}\right) & =\left\langle\phi_{0}, \psi_{I}\left(x_{1} \otimes \cdots \otimes x_{n}\right)\right\rangle \\
& =\left\langle\phi_{0}, A_{I \times\{0\}}\left(x_{1} \otimes \cdots \otimes x_{n}\right) \phi_{0}\right\rangle
\end{aligned}
$$

for all $x \in E$ and all $I \in g_{0}(n)$. This decomposition does not yet suffice to express the operator relations of eq. (3.4). These relations can be expressed in terms of continuity and symmetry-relations for the functionals $T_{I} \in E_{n}^{\prime}$, $I \in g_{0}(n)$.

To this end we denote $g_{0}(0)=\{(0)\}$ and

$$
E(I)=\hat{E}_{0}=\mathbb{C} \quad \text { for } I \in g_{0}(0)
$$

and for $n \geqslant 1$

$$
E(I)=E_{n} \quad \text { for all } I \in \mathcal{F}_{k}(n), k \leq n
$$

and introduce a family of maps

$$
B_{I, J}: E(I) \times E(J) \rightarrow \mathbb{C}
$$

by the following relations:

$$
B_{0,0}\left(a_{0}, b_{0}\right)=\bar{a}_{0} b_{0} \quad \text { for all } a_{0}, b_{0} \in \hat{E}_{0}
$$

For $I \in \mathscr{g}_{0}(n), n \geqslant 1, y \in E(I), a_{0} \in \hat{E}_{0}$

$$
B_{0, I}\left(a_{0}, y\right)=\bar{a}_{0} \cdot T_{I}(y) \text {. }
$$

For $I \in g_{k}(n), J \in g_{k}(m), n, m \geqslant 1, x \in E(I), y \in E(J)$

$$
B_{I, J}(x, y)=\left\langle\psi_{I}(x), \psi_{J}(y)\right\rangle .
$$

Because of (3.2)-(3.4) and (3.11)-(3.14) this family of sesquilinear maps has the following properties: 
(O) $B_{I, J}=0$ if $I \in g_{k}(n), J \in \mathscr{g}_{\ell}(m)$ and $k \neq \downarrow$

(S $\left.\mathrm{S}_{1}\right) B_{I, J}(x, y)=T_{(0) \times I^{*} \dot{x} J}\left(x^{*} \otimes y\right)$ for all $x \in E_{n}, y \in E_{m}$ and for all $I \in g_{k}(n), J \in g_{k}(m), k \leqslant n \wedge m$

$\left(\mathrm{S}_{2}\right) B_{I, H \dot{\times} J}(x, z \otimes y)=B_{H^{*} \dot{\times} I, J}\left(z^{*} \otimes x, y\right)$ for all $x \in E_{n}, z \in E_{r}, y \in E_{m}$ and for all $I \in \mathscr{g}_{k}(n), J \in \mathscr{g}_{\ell}(m), H \in[k, \iota]^{r+1}, k \leq n, \ell \leq m, r \in N$.

For any finite collection of points $a_{I} \in E(I), I \in g_{k}(n), k \leq n$

(P) $0 \leqslant \sum_{k \leq n^{n}, m} \sum_{I \in \mathscr{g}_{k}(n)} \sum_{J \in \mathscr{g}_{k}(m)} B_{I, J}\left(a_{I}, a_{J}\right)$.

Note that the orthogonality relation (O) derives from the orthogonality of different field-sectors, the symmetry-relation (S) is due to the operator relation (3.4) and the hermiticity condition $b$ iii) of a Jacobi-field; and the positivity-condition (P) is a consequence of the definition of $B_{I, J}$ as a scalar product of functions with values in a Hilbert space where the orthogonality relations $(\mathrm{O})$ have been taken into account.

The family $\left\{B_{I, J}\right\}$ of maps introduced in (3.11)-(3.14) can be considered as the components of a nonnegative sesquilinear form $B$ on the space

$$
\begin{aligned}
& \underline{\hat{E}}=\bigoplus_{n=0}^{\infty} \hat{E}_{n} \quad \text { (locally convex direct sum) } \\
& \hat{E}_{0}=\boldsymbol{C} \text { and for } n \geqslant 1 \\
& \hat{E}_{n}=\bigoplus_{n=0}^{n} \underset{I \in \mathscr{g}_{k}(n)}{\bigoplus} E(I)
\end{aligned}
$$

which is again a nuclear barreled space. $B$ is simply defined by

$$
B(\hat{a}, \underline{\hat{b}})=\sum_{k \leq \leq^{n, m}} \sum_{I \in \mathscr{g}_{k}(n)} \sum_{J \in \mathscr{g}_{k}(m)} B_{I, J}\left(a_{I}, b_{J}\right)
$$

if $\underline{\hat{a}}=\left(\hat{a}_{n}\right) \in \underline{\hat{E}}, \hat{a}_{0}=a_{0} \in C, \hat{a}_{n}=\left(a_{I} \mid I \in \mathcal{g}_{k}(n), k \leqslant n\right)$

for $n \geqslant 1$ and the same for $\underline{\hat{b}} \in \underline{\hat{E}}$. Introducing a map $\psi: \underline{\hat{E}} \rightarrow \mathscr{H}$ by

we also have

$$
\psi(\hat{a})=\bigoplus_{k=0}^{\infty} \sum_{n \geqslant k} \sum_{I \in \mathcal{g}_{k}(n)} \psi_{I}\left(a_{I}\right)
$$

$$
B(\hat{a}, \underline{\hat{b}})=\langle\psi(\hat{a}), \psi(\hat{b})\rangle .
$$

It follows that $B$ is a nonnegative continuous sesquilinear form on $\underline{\hat{E}}$.

Its relation to the state $T$ on $\underline{E}$ determined by the Jacobi-field $A$ is conveniently described in terms of the following embedding of $\underline{E}$ into $\underline{E}$ :

$$
\begin{aligned}
& j: \underline{E} \rightarrow \underline{\hat{E}} \\
& j(\underline{x})_{0}=x_{0}, j(\underline{x})_{n}=\hat{x}_{n}=\left(x_{I}=x_{n} \mid I \in \mathcal{g}_{k}(n), k \leq n\right), \forall \underline{x} \in \underline{E} .
\end{aligned}
$$


If we observe now for $n, m=1,2, \cdots$

$$
g_{0}(n+m)=\left\{\{0\} \times I^{*} \dot{\times} J \mid I \in g_{k}(n), J \in g_{k}(m), 0 \leq k \leq n \wedge m\right\}
$$

we obtain for all $\underline{x}, \underline{y} \in \underline{E}$ using (3.18), (3.15), and (3 9)

$$
\begin{aligned}
& B(j(\underline{x}), j(\underline{y}))=\sum_{k \leq^{n \wedge m}} \sum_{I \in \mathscr{g}_{k}(n)} \sum_{J \in \mathscr{g}_{k}(m)} B_{I, J}\left(x_{I}, y_{J}\right) \\
& =\sum_{k \leq^{n \wedge m}} \sum_{I \in \mathcal{I}_{k}(n)} \sum_{J \in \mathcal{G}_{k}(m)} T_{\{0\} \times I^{*} \dot{\times} J}\left(x_{I}^{*} \otimes y_{J}\right) \\
& =\sum_{n, m}\left(\sum_{k S^{n \wedge m}} \sum_{I \in \mathscr{g}_{k}(n)} \sum_{J \in \mathscr{g}_{k}(m)} T_{\{0\} \times I^{*} \dot{\times} J}\right)\left(x_{n}^{*} \otimes y_{m}\right) \\
& =\sum_{n, m}\left(\sum_{H \in g_{0}(n+m)} T_{H}\right)\left(x_{n}^{*} \otimes y_{m}\right) \\
& =\sum_{n, m} T_{n+m}\left(x_{n}^{*} \otimes y_{m}\right)
\end{aligned}
$$

e.g.

$$
B(j(\underline{x}), j(\underline{y}))=T\left(\underline{x}^{*} \cdot \underline{y}\right) \text { for all } \underline{x}, \underline{y} \in \underline{E} .
$$

Next the density property (3.8) is expressed in terms of this sesquilinear form $B$. This is achieved by introducing first a certain subspace of $\underline{E}$ :

$$
\hat{E}_{0}=\left\{\hat{\hat{b}} \in \underline{\hat{E}} \mid \begin{array}{l}
\hat{b}_{n}=\left(b_{I} \mid I \in \mathcal{g}_{k}(n), k \leq n\right) \\
b_{I}=0 \text { for all } I \in \mathcal{g}_{k}(n), k \leq n-1, b_{I} \in E^{\otimes n} \text { for } I \in \mathcal{g}_{n}(n)
\end{array}\right\} .
$$

By (3.19) the density property (3.8) just says that

$$
\psi\left(\underline{E}_{0}\right) \text { is dense in the statespace of the Jacobi-field } A .
$$

In terms of $B$ this reads as follows if we use $\left(3.18^{\prime}\right)$ :

For all $\underline{\hat{a}} \in \underline{\hat{E}}: \inf \left\{B(\underline{\hat{a}}-\underline{\hat{b}}, \underline{\hat{a}}-\underline{\hat{b}}) \mid \underline{\hat{b}} \in \underline{\hat{E}}_{0}\right\}=0$

Denote by $\mathscr{B}^{+}(\underline{\hat{E}})$ the cone of all non-negative (separately) continuous sesquilinear forms on $\underline{\hat{E}}$ and then by $\mathscr{B}_{0}^{+}(\underline{\hat{E}})$ the subset of those $B$ satisfying the normalization condition (3.12) and the orthogonality relation (O). Finally we define

$$
\mathscr{B}_{J}^{+}(\underline{\hat{E}})=\left\{B \in \mathscr{B}_{0}^{+}(\underline{\hat{E}}) \mid B \text { satisfies }(\mathrm{S}) \text { and }(\mathrm{D})\right\}
$$

and arrive at

Proposition 3.1. For every Jacobi-state $T$ on $\underline{E}$ there is $B=B^{T} \in \mathscr{B}_{J}^{+}(\underline{\hat{E}})$ such that for all $\underline{x}, \underline{v} \in \underline{E}$

$$
T\left(\underline{x}^{*} \cdot \underline{y}\right)=B(j(\underline{x}), j(\underline{y}))
$$

holds. This representation of $T \in \mathcal{E}_{J}(\underline{E})$ is called the Jacobi-decomposition of the state $T$. 
Remark 3.1. By this definition a state $T$ on $\underline{E}$ admits a Jacobi-decomposition iff the components $T_{n}$ of $T$ admit the decomposition 3.9 with the functionals $T_{I} \in E_{n}^{\prime}$ for $I \in g_{0}(n)$, having the properties expressed by (3.15) and (D).

\section{III.2. Functional characterization of Jacobi-states.}

Proposition 3.1 says that every Jacobi-state admits a Jacobi-decomposition. Here we show that conversely every state on $\underline{E}$ which admits a Jacobidecomposition is a Jacobi-state and that this decomposition is unique.

\section{Proposition 3.2.}

a) With every $B \in \mathscr{B}_{0}^{+}(\underline{\hat{E}})$ there is canonically associated a separable Hilbert space $\mathcal{H}$ and a continuous linear map $\psi: \underline{\hat{E}} \rightarrow \mathcal{H}$ with dense range such that

$$
B(\underline{\hat{a}}, \underline{\hat{b}})=\langle\psi(\hat{a}), \psi(\underline{\hat{b}})\rangle \quad \text { for all } \underline{\hat{a}}, \underline{\hat{b}} \in \underline{\hat{E}}
$$

b) The associated Hilbert space has the following orthogonal decomposition

$$
\mathscr{H}=\bigoplus_{k=0}^{\infty} \mathscr{H}^{k}
$$

with

$$
\mathscr{H}^{0}=\boldsymbol{C} \psi(j(1))
$$

and $\quad \mathscr{H}^{k}=$ closure of $\mathscr{D}^{k}$ in $\mathscr{H}$ for $k \geqslant 1$

$$
\mathscr{D}^{k}=\operatorname{lin} \bigcup_{n \geq k} \bigcup_{I \in \mathscr{g}_{k}(n)} \psi_{I}(E(I))
$$

where

$$
\psi_{I}=\psi \uparrow E(I)
$$

Proof. Part a) is quite standard. Therefore we only prove b). By (3.25) we have for all $I \in \mathscr{g}_{k}(n), J \in \mathscr{g}_{\ell}(m), a_{I} \in E(I)$, and $b_{J} \in E(J)$

$$
B_{I, J}\left(a_{I}, b_{J}\right)=\left\langle\psi_{I}\left(a_{I}\right), \psi_{J}\left(b_{J}\right)\right\rangle
$$

and therefore $\psi_{I}(E(I))$ is orthogonal to $\psi_{J}(E(J))$ whenever $k \neq \downarrow$ according to (O). This implies that $\mathscr{D}^{k}$ as defined by (3.28) is orthogonal to $\mathscr{D}^{l}$ for $k \neq l$. By construction we know $\psi(\hat{E})$ to be dense in $\mathscr{H}$, and we have

$$
\psi(\underline{\hat{E}})=\bigoplus_{k=0}^{\infty}\left(\sum_{n \geqslant k} \sum_{I \in \mathcal{G}_{k}(n)} \psi_{I}(E(I))\right) \subseteq \bigoplus_{k=0}^{\infty} \mathscr{D}^{k}
$$

This proves $b$ ).

The next proposition characterizes the symmetry conditions for $B \in \mathscr{B}_{0}^{+}(\underline{\hat{E}})$.

Proposition 3.3. $B \in \mathscr{B}_{0}^{+}(\underline{\hat{E}})$ satisfies the symmetry relations (S) iff in the Hilbert space realization of $B$ according to Proposition 3.2 the following holds: 
For $k, \ell=0,1,2, \cdots, r=1,2, \cdots$ and $H \in[k, \zeta]^{r+1}$ there is $A_{H} \in \mathcal{L}_{s c}\left(E_{r}, L\left(\mathscr{D}^{l}\right.\right.$, $\left.\mathscr{D}^{k}\right)$ ) such that
i) $A_{I \times\{0\}}(x) \psi(j(1))=\psi_{I}(x) \quad$ for all $x \in E_{r}, I \in g_{k}(r), k \leq r$
ii) $A_{H}(x) \psi_{J}(y)=\psi_{H \dot{\times} J}(x \otimes y) \quad$ for all $x \in E_{r}, J \in g_{\ell}(m), y \in E_{m}$
iii) $\left(A_{H}(x)\right)^{*} \supseteq A_{H^{*}}\left(x^{*}\right) \quad$ for all $x \in E_{r}$.

Proof. Given the Hilbert space realization of $B$ according to Proposition 3.2, the symmetry relations $\left(\mathrm{S}_{1}\right)$ and $\left(\mathrm{S}_{2}\right)$ are expressed in terms of the $\left\{\psi_{1}\right\}$ by

$$
\begin{aligned}
& \left\langle\psi_{I}(x), \psi_{J}(y)\right\rangle=\left\langle\psi_{0}, \psi_{\{0\} \times I^{\prime} \dot{x} J}\left(x^{*} \otimes y\right)\right\rangle \\
& \text { for all } I \in g_{k}(n), J \in g_{k}(m), x \in E_{n}, y \in E_{m}
\end{aligned}
$$

where $\psi_{0}=\psi(j(1))$ and

$$
\left\langle\psi_{I}(z), \psi_{H \dot{x} J}(x \otimes y)\right\rangle=\left\langle\psi_{H^{*} \dot{x} J}\left(x^{*} \otimes z\right), \psi_{J}(y)\right\rangle
$$

$$
\text { for all } I \in \mathcal{G}_{k}(n), J \in \mathscr{g}_{l}(m), H \in[k, \iota]^{r+1}, x \in E_{r}, y \in E_{m}, z \in E_{n} \text {. }
$$

By $\left(\mathrm{S}_{2}^{\prime}\right) \quad \psi_{J}(y)=0$ implies $\psi_{H \dot{ }}(x \otimes y) \in \mathscr{D}^{k} \cap\left(\mathscr{D}^{k}\right)^{\perp}=\{0\}$. Therefore for each $x \in E_{r}$, each $H \in[k, \ell]^{r+1}$ a linear operator $A_{H}(x): \mathscr{D}^{l} \rightarrow \mathscr{D}^{k}$ is well defined by Eq. (3.31) and because of $\left(\mathbf{S}_{2}^{\prime}\right)$ this operator satisfies the hermiticity condition (3.32).

Every $H \in[k, O]^{r+1}$ has a unique representation as $H=I x\{0\}$ with $I \in g_{k}(r)$, $k \leq r$; thus $A_{I \times\{0\}}(x)$ is well defined by (3.30) and $\left(\mathrm{S}_{1}\right)$ implies that this definition is consistent with the above definition of $A_{H}$ and that in particular (3.32) holds for this case.

The strong continuity of $A_{H}$ thus defined follows directly from the continuity of $\psi_{H \dot{\times} J}: E_{r} \otimes E_{m} \rightarrow \mathcal{H}^{k}$ and the continuity of $E_{r} \ni x \rightarrow x \otimes y \in E_{r} \otimes E_{m}$ for fixed $y \in E_{m}$. As the converse is obvious Proposition 3.3 is proved.

After this preparations the functional characterization of Jacobi-states is simple. Suppose $B \in \mathscr{B}_{J}^{+}(\underline{\hat{E}})$ to be given. Then construct $\mathscr{H}$ and $\mathscr{D}^{k}$ and $\mathscr{H}^{k}$ according to Proposition 3.2 and

$$
\mathscr{D}=\bigoplus_{k=0}^{\infty} \mathscr{D}^{k} \quad \text { (direct sum) }
$$

Next define $A_{i j}$ for $|i-j| \leq 1$ by Proposition 3.3 by taking $H=\{i, j\} \in[i, j]^{1}$ there. Then we know $A_{i j} \in \mathcal{L}_{s c}^{h}\left(E, L\left(\mathscr{D}^{j}, \mathscr{D}^{i}\right)\right)$ and thus $A \in \mathcal{L}_{s c}^{h}(E, L(\mathscr{D}, \mathscr{D}))$ is well defined by Eq. iv) of Definition 1.2.

If we now calculate the subspaces $\mathscr{H}_{(n)}$ associated with $A$ according I.1 and introduce the subspace $\mathscr{H}_{k}$ by Eq. (1.3) such that 


$$
\begin{array}{ll} 
& \mathscr{H}_{(n)}=\bigoplus_{k=0}^{n} \mathscr{H}_{k} \\
\text { we see } & \mathscr{H}_{k}=\overline{\mathscr{D}}_{k} \quad(\text { closure in } \mathscr{H}) \\
\text { where } & \mathscr{D}_{k}=A_{k k-1}(E) \cdots A_{21}(E) A_{10}(E) \psi_{0}, \psi_{0}=\psi(j(1)) .
\end{array}
$$

Because of the density condition (D) for $B$ the direct sum $\bigoplus_{k=0}^{\infty} \mathscr{D}_{k}$ is known to be dense in $\mathscr{H}$. As obviously $\mathscr{H}_{k} \subseteq \mathscr{H}^{k}$ it follows

$$
\begin{array}{ll}
\mathscr{H}=\bigoplus_{k=0}^{\infty} \mathscr{H}_{k}=\bigoplus_{k=0}^{\infty} \mathscr{H}^{k} & \text { and thus } \\
\mathscr{H}^{k}=\mathscr{H}_{k} & k=0,1,2, \cdots .
\end{array}
$$

This shows that

i) $\psi_{0}$ is cyclic for $\{A(x) \mid x \in E\}$.

Thus $A$ is a field over $E$ in $\mathscr{A}$.

ii) $\mathscr{H}^{k}=\mathscr{H}_{k}$ are the field-sectors of $A$.

Thus $A$ is a Jacobi-field with domain $\mathscr{D}$ and cyclic vector $\psi_{0}$.

Now we define a Jacobi-state $T$ on $\underline{E}$ as the sequence of $n$-point-functionals of this field $A$. By the same calculation as earlier it follows that this Jacobi-state $T$ is related to the sesquilinear form $B \in \mathscr{B}_{J}^{+}(\underline{\underline{E}})$ by Equation (3.22). Thus taking Proposition 3.1 into account we have proved

Proposition 3.4. Suppose the basic space E satisfies (H). Then a state $T$ on $\underline{\hat{E}}$ is a Jacobi-state iff $T$ is of the form

$$
T\left(x^{*} \circ \underline{y}\right)=B(j(x), j(\underline{y})) \quad \text { for all } \underline{x}, \underline{y} \in \underline{E}
$$

with some $B \in \mathscr{B}_{J}^{+}(\hat{E})$.

Naturally in this context the question arises whether $B \in \mathscr{B}_{J}^{+}(\underline{\hat{E}})$ is unique in (3.22) or not. This will be answered affirmatively after we have investigated some important consequences of the density condition (D) for the structure of $B$.

By its definition the $k$-field sector $\mathscr{H}_{k}$ associated with $B \in \mathscr{B}_{J}^{+}(\underline{\underline{E}})$ according to Proposition 3.4 is generated by vectors of the form

$$
\psi_{I(k)}(x), x \in E^{\otimes k}, I(k)=(k, k-1, \cdots, 2,1)
$$

for $k \geq 1$ and by $\psi_{0}=\psi(j(1))$ for $k=0$. This implies for $k=1,2, \cdots$ that there is a sequence

$$
\left\{e_{j}^{k} \mid j \in N\right\} \subset E^{\otimes k}
$$

depending only on the component $B_{I(k), I(k)}$ of $B$ such that

$$
\left\{\psi_{I(k)}\left(e_{j}^{k}\right) \mid j \in N\right\}
$$

is an orthonormal basis of $\mathscr{H}_{k}=\mathscr{H}^{k}$. Applying the completeness relations 
for this basis to

we arrive at

$$
B_{I, J}(x, y)=\left\langle\psi_{I}(x), \psi_{J}(y)\right\rangle
$$

$$
B_{I, J}(x, y)=\sum_{J}^{\infty} B_{I, I(k)}\left(x, e_{J}^{k}\right) B_{I(k), J}\left(e_{j}^{k}, y\right)
$$

c.g.

$$
B_{I, J}=B_{I, I(k)}\left(\mathbb{k} B_{I(k), J} \quad \text { for all } I \in \mathcal{J}_{k}(n), J \in \mathcal{I}_{k}(m)\right.
$$

where the kernel $K_{k}$ of the "convolution" $\left(\mathcal{B}\right.$ )only depends on $B_{I(k), I(k)}$.

In particular we have for $I \in \mathscr{g}_{0}(n), J \in \mathscr{g}_{0}(m), x \in E_{n}$, and $y \in E_{m}$ by $\left(\mathrm{S}_{1}\right)$ and normalization

$$
B_{I, J}(x, y)=T_{I}\left(x^{*}\right) T_{J}(y)
$$

More generally it follows for $I \in \mathcal{g}_{k}(n), J \in g_{0}(n), x \in E_{n}, y \in E_{m}$

$$
\psi_{I \times J}(x \otimes y)=\psi_{I}(x) T_{J}(y) .
$$

Lemma 3.5. The components $B_{I, J}$ of $B \in \mathscr{B}_{J}^{+}(\underline{\hat{E}})$ satisfy the Equations (3.37) and (3.38). In the Hilbert space realization of $B$ according to Proposition (3.2) the factorization property (3.39) holds.

As the most important consequence of relations (3.37)-(3.39) we prove that $B \in \mathscr{B}_{J}^{+}(\underline{\hat{E}})$ is already determined by its values on $j(\underline{E}) \subset \underline{\hat{E}}$ :

Proposition 3.6. Suppose $B, B^{\prime} \in \mathscr{B}_{J}^{+}(\underline{\hat{E}})$ satisfy

$$
B(j(\underline{x}), j(\underline{y}))=B^{\prime}(j(\underline{x}), j(\underline{y})) \quad \underline{x}, \underline{y} \in \underline{E}
$$

then $B=B^{\prime}$.

Proof. a) The proof is done by showing by induction that $G_{N}$ holds for $N=0,1,2, \cdots$ where $G_{N}$ is the following statement:

$$
B_{I, J}=B_{I, J}^{\prime} \quad \text { for all } I \in \mathcal{g}_{k}(n), J \in \mathcal{g}_{k}(m) ; k \leq n \wedge m ; n, m \leq N .
$$

For $N=0$ this holds by normalization. So we start with $N=1$.

By definition of the embedding $j$ Equation (3.40) reads for all $a_{0}, b_{0} \in \mathbb{C}$ and all $a_{1}, b_{1} \in E_{1}$ :

$$
\begin{aligned}
& \bar{a}_{0} b_{0}+\bar{a}_{0} T_{(0)}\left(b_{1}\right)+T_{(0)}\left(a_{1}^{*}\right) b_{0}+T_{(0)}\left(a_{1}^{*}\right) T_{(0)}\left(b_{1}\right)+B_{(1),(1)}\left(a_{1}, b_{1}\right) \\
= & \bar{a}_{0} b_{0}+\bar{a}_{0} T_{(0)}^{\prime}\left(b_{1}\right)+T_{(0)}^{\prime}\left(a_{1}^{*}\right) b_{0}+T_{(0)}^{\prime}\left(a_{0}^{*}\right) T_{(1)}^{\prime}\left(b_{1}\right)+B_{(1),(1)}^{\prime}\left(a_{1}, b_{1}\right) .
\end{aligned}
$$

This yields immediately

$$
\begin{array}{ll}
T_{(0)}=T_{(0)}^{\prime}=T_{1} \in E_{1}^{\prime} & \text { a) } \\
B_{(1),(1)}=B_{(1),(1)}^{\prime} & \text { b) }
\end{array}
$$


and $\left(G_{1}\right)$ follows.

The symmetry relations (S) and (3.41) imply further more

$$
\begin{aligned}
& T_{(01)}=B_{(0),(01)}=B_{(1),(1)}=B_{(1),(1)}^{\prime}=B_{(0),(01)}^{\prime}=T_{(01)}^{\prime} \\
& \text { and } \quad B_{(01),(0)}=B_{(01),(0)}^{\prime} .
\end{aligned}
$$

b) Now we prove that if $\left(G_{N}\right)$ holds for some $N \geq 1$ then it also holds for $N$ +1 . The induction hypothesis thus is

$$
B_{I, J}=B_{I, J}^{\prime} \quad \text { for all } I \in \mathcal{g}_{k}(n), J \in g_{k}(m) ; k \leq n \wedge m, n, m \leq N .
$$

First consider the cases $n=N+1$ and $m \leq N-1$. Observe that $I \in \mathcal{G}_{k}(n+1)$ can be written as $I=(k) \times I^{\prime}$ with $I^{\prime} \in g_{\ell}(N)$ and $\ell \leq k+1 \leq m+1 \leq N$. So the symmetry relation $\left(\mathbf{S}_{2}\right)$ implies

$$
B_{I, J}=B_{I^{\prime},(k) \times J}=B_{I^{\prime},(k) \times J}^{\prime}=B_{I, J}^{\prime}
$$

where the second equality holds because $(+)$ applies $\left((k) \times J \in g_{k}(m+1)\right.$ and $m+1 \leq N)$.

Now we consider the case $n=N+1$ and $m=N$. For $I \in \mathscr{g}_{k}(N+1), J \in \mathscr{g}_{k}(N)$ and $k=0,1, \cdots, N$ Eq. (3.37) yields

$$
B_{I, J}=B_{I, I(k)}(k) B_{I(k), J} \cdot
$$

As $k \leq N(+)$ applies: $\quad B_{I(k), J}=B_{I(k), J}^{\prime}$ for all $J \in \mathcal{g}_{k}(N)$ and $K_{k}=K_{k}^{\prime}$.

If now $k \leq N-1$ we write $I \in g_{k}(N+1)$ as $I=(k) \times I^{\prime}$ with $I^{\prime} \in \mathcal{g}_{\ell}(N)$ and $\ell \leq N$ and obtain by $\left(\mathrm{S}_{2}\right)$ and $(+)$ :

$$
B_{I, I(k)}=B_{I^{\prime},(k) \times I(k)}=B_{I^{\prime},(k) \times I(k)}^{\prime}=B_{I, I(k)}^{\prime} .
$$

By $\left(3.37^{\prime}\right)$ and the preceding observations it follows

$$
B_{I, J}=B_{I, J}^{\prime} \text { for all } I \in \mathscr{g}_{k}(N+1), J \in \mathscr{g}_{k}(N), k \leq N-1 .
$$

Next we consider $n=N+1, m=N$ and $k=N$. For all $x \in E_{N+1}$ and $y \in E_{N}$ Eq. (3.40) reads in terms of components

$$
\sum_{k=0}^{N} \sum_{I \in \mathcal{g}_{k}(N+1)} \sum_{J \in \mathscr{g}_{k}(N)} B_{I, J}(x, y)=\sum_{k=0}^{N} \sum_{J \in \mathcal{I}_{k}(N+1)} \sum_{I \in \mathcal{g}_{k}(N)} B_{I, J}^{\prime}(x, y) .
$$

Thus (3.44) implies

$$
\sum_{I \in \mathcal{I}_{N}(N+1)} B_{I, I(N)}(x, y)=\sum_{I \in \mathcal{g}_{N}(N+1)} B_{I, I(N)}^{\prime}(x, y) .
$$

In the decomposition of $I$,

$$
I=(N) \times I^{\prime},
$$


there is only one possibility with $I^{\prime} \in \mathcal{G}_{N}(N)$. All the others have $I^{\prime} \in \mathcal{G}_{N-1}(N)$. For these cases we use again $\left(\mathrm{S}_{2}\right),(+)$, and (3.37):

$$
\begin{aligned}
& \left.B_{(N) \times I^{\prime}, I(N)}=B_{I^{\prime},(N-1) \times I(N)}=B_{I^{\prime}, I(N-1)} N-1\right) B_{I(N-1),(N-1) \times I(N)} \\
= & \left.B_{I^{\prime}, I(N-1)}^{\prime} N-1\right) B_{I(N-1),(N-1) \times I(N)}^{\prime}
\end{aligned}
$$

and thus

$$
B_{(N) \times I^{\prime}, I(N)}=B_{(N) \times I^{\prime}, I(N)}^{\prime} \quad \text { for all } I^{\prime} \in g_{N-1}(N)
$$

and therefore by (3.45) we get

$$
B_{(N) \times I(N), I(N)}=B_{(N) \times I(N), I(N)}^{\prime} .
$$

By hermiticity and the symmetry relations (S) this shows altogether

$$
\begin{aligned}
B_{I, J}=B_{I, J}^{\prime} \text { for } I & \in \mathscr{g}_{k}(n), J \in \mathscr{g}_{k}(m), k \leq n \wedge m \\
& n \leq N \text { and } m \leq N+1 \text { or } n \leq N+1 \text { and } m \leq N .
\end{aligned}
$$

It remains to consider the case $n=n i=N+1$. For $k \leq N$ we apply (3.37) and use (3.48) to conclude for $I, J \in \mathscr{g}_{k}(N)$

$$
B_{I, J}=B_{I, I(k)}\left(\mathcal{k} B_{I(k), J}=B_{I, I(k)}^{\prime}(k)^{\prime} B_{I(k), J}^{\prime}=B_{I, J}^{\prime}\right.
$$

For $x, y \in E_{N+1}$ assumption (3.40) reads in terms of components

$$
\sum_{k=0}^{N+1} \sum_{I, J \in \mathscr{g}_{k}(N+1)} B_{I, J}(x, y)=\sum_{k=0}^{N+1} \sum_{I, J \in g_{k}(N+1)} B_{I, J}^{\prime}(x, y) \text {. }
$$

Thus by (3.49) we conclude

$$
B_{I(N+1), I(N+1)}=B_{I(N+1), I(N+1)}^{\prime} .
$$

Equations (3.48)-(3.50) together thus say that $\left(G_{N+1}\right)$ holds, and this then proves $B=B^{\prime}$.

Propositions 3.4 and 3.6 yield the main result of this section.

Theorem 3.7. Suppose the basic space $E$ satisfies $(\mathrm{H})$. Then a state $T$ on $\underline{E}$ is a Jacobi-state iff $T$ is of the form

$$
T\left(\underline{x}^{*} \cdot \underline{y}\right)=B(j(x), j(\underline{y})) \quad \underline{x}, \underline{y} \in \underline{E}
$$

where $B \in \mathscr{B}_{J}^{+}(\underline{\hat{E}})$. The correspondance $T \rightarrow B$ defined by this equation is one-toone.

\section{§IV. Some Properties of Jacobi-States}

IV.1. Convexity and order properties.

The results of the last section show that there is a one-to-one correspond- 
ence between Jacobi-states $T$ and sesquilinear forms $B$ on $\underline{\hat{E}}$ belonging to $\mathscr{B}_{J}^{+}(\underline{\hat{E}})$. Therefore we can study $\mathcal{E}_{J}(\underline{E})$ by studying $\mathscr{B}_{J}^{+}(\underline{\hat{E}})$. This turns out to be more efficient because the characterization of sesquilinear forms to belong to $\mathscr{B}_{J}^{+}(\underline{\hat{E}})$ only refers to properties of functionals and not to properties of an algebra of unbounded operators as it is the case for $\mathcal{E}_{J}(\underline{E})$.

By definition elements in $\mathscr{B}_{0}^{+}(\underline{\hat{E}})$ are distinguished from arbitrary continuous nonnegative sesquilinear forms on $\underline{\hat{E}}$ by the two linear constraints of normalization and orthogonality whereas elements in $\mathscr{B}_{J}^{+}(\underline{\hat{E}})$ are distinguished from elements in $\mathscr{B}_{0}^{+}(\hat{E})$ by the fact that they have to verify in addition the linear constraint (S) of symmetry and the non-linear constraint (D) of "density". This implies that it is fairly easy to control various "stability-properties" within $\mathscr{B}_{0}^{+}(\underline{\hat{E}})$ but not in $\mathscr{B}_{J}^{+}(\underline{\hat{E}})$ because of the non-linear condition (D). In this respect the following property of $\mathscr{B}_{J}^{+}(\underline{\hat{E}})$ to be order convex in

$$
\mathscr{B}_{0 s}^{+}(\hat{E})=\left\{B \in \mathscr{B}_{0}^{+}(\underline{\hat{E}}) \mid B \text { satisfies }(\mathrm{S})\right\}
$$

is of some importance. Here the natural order structure of $\mathscr{B}^{+}(\underline{\hat{E}})$ is used, e.g.

$$
B_{1}, B_{2} \in \mathscr{B}^{+}(\underline{\hat{E}}), B_{1} \leq B_{2} \text { iff } B_{1}(\underline{\hat{a}}, \underline{\hat{a}}) \leq B_{2}(\underline{\hat{a}}, \underline{\hat{a}}), \underline{\hat{a}} \in \underline{\hat{E}} .
$$

For $B \in \mathscr{B}^{+}(\underline{\hat{E}})$ we denote by $q_{B}$ the following seminorm on $\underline{\hat{E}}$ :

$$
q_{B}(\hat{a})=B(\underline{\hat{a}}, \underline{\hat{a}})^{1 / 2} .
$$

By Theorem III.5.1 of [11] $q_{B}$ is continuous.

For a continuous seminorm $q$ on $\underline{\hat{E}}$ and a subset $M \subset \underline{\hat{E}}$ we denote by $\bar{M}^{q}$ the closure of this set (in $\underline{\hat{E}}$ ) with respect to $q$. With these notations we obtain a simple reformulation of the density property (D).

Lemma $4 . \mathcal{H}_{\text {. For }} B \in \mathscr{B}_{0}^{+}(\hat{E})$ the following statements are equivalent:

i) $B$ satisfies condition (D)

ii) $\underline{\hat{E}}=\overline{\hat{\hat{E}}}_{0}^{q B}$

iii) $B(\underline{\hat{a}}, \underline{\hat{a}})=\sup _{\underline{\hat{b}} \in \underline{\hat{E}}_{0}}\left\{|B(\underline{\hat{a}}, \underline{\hat{b}})|^{2} B(\underline{\hat{b}}, \underline{\hat{b}})^{-1}\right\} \quad$ for all $\underline{\hat{a}} \in \underline{\hat{E}}$

where the quotient is defined to be zero if $B(\underline{\hat{b}}, \underline{\hat{b}})=0$.

Proof. The equivalence of i) and ii) is just a matter of definitions. By positivity of $B$ we know

$$
|B(\underline{\hat{a}}, \underline{\hat{b}})|^{2} \leq B(\underline{\hat{a}}, \underline{\hat{a}}) \cdot B(\underline{\hat{b}}, \underline{\hat{b}}) .
$$

Therefore the supremum exists. In the Hilbert space realization of $B$ accord- 
ing to Proposition 3.2 this supremum equals $\|P \psi(\hat{a})\|^{2}$ where $P$ is the projection from the Hilbert space $\mathscr{H}$ associated with $B$ onto the subspace generated by $\psi\left(\underline{\hat{E}}_{0}\right)$. Thus (4.4) says $\|\psi(\underline{\hat{a}})\|^{2}=\|\boldsymbol{P} \psi(\underline{\hat{a}})\|^{2}$ for all $\underline{\hat{a}} \in \underline{\hat{E}}$ and therefore iii) is equivalent to (D).

The announced order property is an immediate consequence of condition (D) directly or relation (4.3). It reads

Corollary 4.2. Suppose $B \in \mathscr{B}_{J}^{+}(\underline{\hat{E}})$ to be given. If for $B^{\prime} \in \mathscr{B}_{0 s}^{+}(\underline{\hat{E}})$ there is $\lambda>0$ such that $B^{\prime} \leq \lambda B$ then $B^{\prime} \in \mathscr{B}_{J}^{+}(\underline{\hat{E}})$.

Now we come to discuss convexity of $\mathcal{E}_{J}(\underline{E})$, e.g. of $\mathscr{B}_{J}^{+}(\underline{\hat{E}})$. The set $\mathscr{B}_{0 s}^{+}(\underline{\hat{E}})$ is obtained from the convex cone $\mathscr{B}^{+}(\underline{\hat{E}})$ by imposing three linear constraints. Thus $\mathscr{B}_{0 s}^{+}(\underline{\hat{E}})$ is convex. The problem arises when the density condition (D) is taken into account as the convex combination of two functions having infimum zero need not to have zero as its infimum.

Therefore within $\mathscr{B}_{J}^{+}(\underline{\hat{E}})$ we are confined to consider convexity-classes of $B$ $\in \mathscr{B}_{J}^{+}(\underline{\hat{E}})$ :

$$
\mathcal{K}_{B}=\left\{B^{\prime} \in \mathscr{B}_{J}^{+}(\underline{\hat{E}}) \mid B_{\lambda}=\lambda B+(1-\lambda) B^{\prime} \in \mathscr{B}_{J}^{+}(\underline{\hat{E}}) \text { for all } \lambda \in[0,1]\right\}
$$

which are characterized by

Lemma 4.3. Suppose $B, B^{\prime} \in \mathscr{B}_{J}^{+}(\underline{\hat{E}})$ to be given. Then

$$
B^{\prime} \in \mathcal{K}_{B} \text { iff for all } \underline{\hat{a}} \in \underline{\hat{E}}: \inf \left\{\left(B+B^{\prime}\right)(\underline{\hat{a}}-\underline{\hat{b}}, \underline{\hat{a}}-\underline{\hat{b}}) \mid \underline{b} \in \underline{\hat{E}}_{0}\right\}=0 \text {. }
$$

Proof. If $B^{\prime} \in \mathcal{K}_{B}$ then for all $\lambda \in[0,1] B_{\lambda}=\lambda B+(1-\lambda) B^{\prime}$ satisfies (D). Thus for $0<\lambda<1$ a simple estimate implies (4.6). The converse is implied by Corollary 4.2 and the observation that $B_{\lambda} \leq B+E^{\prime}$.

The question arises whether for a given $B \in \mathscr{B}_{J}^{+}(\underline{\hat{E}})$ the convexity-class is bigger than $\{B\}$ or not. But this is already answered by Corollary 4.2 and Lemma 4.3 in the following way:

$$
\left\{B^{\prime} \in \mathscr{B}_{0 s}^{+}(\underline{\hat{E}}) \mid \text { there is } \lambda>0 \text { such that } B^{\prime} \leq \lambda B\right\} \subset \mathcal{K}_{B} .
$$

The following proposition shows that there is at least one convexity class which is fairly big. It is that "containing" the set of all bounded Jacobi-states.

Proposition 4.4. The set of all bounded Jacobi-states is convex.

Proof. a) If $T^{i} \in \mathcal{E}_{J b}(\underline{E})$ are given we can suppose that these states are the sequences of $n$-point-functionals of two bounded Jacobi-fields $A^{i}$ in the Hilbert spaces

$$
\mathscr{H}^{i}=\bigoplus_{k=1}^{\infty} \mathscr{H}_{k}^{i} \quad i=1,2
$$


with cyclic unit vector $\phi_{0}^{i}, \mathcal{H}_{0}^{i}=\mathbb{C} \phi_{0}^{i}$. For $0<\lambda<1$ we consider

$$
T=\lambda T^{1}+(1-\lambda) T^{2} \in \mathcal{E}(\underline{E})
$$

and want to check whether $T$ is a Jacobi-state or not.

Define

$$
\phi_{0}=\left(\begin{array}{cc}
\lambda^{1 / 2} & \phi^{1} \\
\lambda^{1 / 2} & \phi^{2}
\end{array}\right) \in\left(\begin{array}{c}
\mathscr{H}^{1} \\
\mathscr{H}^{2}
\end{array}\right), \lambda^{\prime}=1-\lambda
$$

and

$$
A(x)=\left(\begin{array}{cc}
A^{1}(x) & 0 \\
0 & A^{2}(x)
\end{array}\right) \text { in }\left(\begin{array}{c}
\mathscr{H}^{1} \\
\mathscr{H}^{2}
\end{array}\right), x \in E
$$

on $\left(\begin{array}{l}\mathscr{D}^{1} \\ \mathscr{D}^{2}\end{array}\right), \mathscr{D}^{i}=\bigoplus_{k=0}^{\infty} \mathscr{H}_{k}^{i} \quad$ (direct sum).

We get for all $x_{i} \in E$

$$
A\left(x_{1}\right) \cdots A\left(x_{n}\right) \phi_{0}=\left(\begin{array}{lll}
A^{1}\left(x_{1}\right) & \cdots & A^{1}\left(x_{n}\right) \phi_{0}^{1} \\
A^{2}\left(x_{1}\right) & \cdots & A^{2}\left(x_{n}\right) \phi_{0}^{2}
\end{array}\right)
$$

and thus for all $x \in E$ and all $n$

$$
\begin{aligned}
\left\langle\phi_{0}, A\left(x_{1}\right) \cdots A\left(x_{n}\right) \phi_{0}\right\rangle & =\lambda T_{n}^{1}\left(x_{1} \otimes \cdots \otimes x_{n}\right)+\lambda^{\prime} T_{n}^{2}\left(x_{1} \otimes \cdots \otimes x_{n}\right) \\
& =T_{n}\left(x_{1} \otimes \cdots \otimes x_{n}\right) .
\end{aligned}
$$

Therefore it suffices to show that $A$ is (equivalent to) a Jacobi-field.

b) Denote by $\mathscr{H}_{(n)}$ the closure of the linear span of $\left\{A\left(x_{1}\right) \cdots A\left(x_{r}\right) \phi_{0} \mid x_{i} \in E\right.$, $r \leq n\}$ in $\left(\begin{array}{l}\mathscr{H}^{1} \\ \mathscr{H}^{2}\end{array}\right)$ and by $\mathscr{H}$ the closure of $\bigcup_{n=0}^{\infty} \mathscr{H}_{(n)}$ in $\left(\begin{array}{l}\mathscr{H}^{1} \\ \mathscr{H}^{2}\end{array}\right)$. Thus $\mathscr{H}_{(n)}$ is a subspace of $\left(\begin{array}{l}\mathcal{H}_{(n)}^{1} \\ \mathcal{H}_{(n)}^{2}\end{array}\right), n=0,1,2, \ldots$ and $\mathscr{H}$ a subspace of $\left(\begin{array}{l}\mathscr{H}^{1} \\ \mathscr{H}^{2}\end{array}\right)$. By this construction the unit vector $\phi_{0}$ is cyclic for $\{A(x) \mid x \in E\}$ in $\mathscr{H}$. Using (4.9) and the corresponding properties of the $A^{i}$ it easily follows that $A$ is hermitian and strongly continuous.

Next we define

$$
\mathscr{H}_{0}=\mathscr{H}_{(0)}=\boldsymbol{C} \phi_{0}
$$

and for $n=0,1,2, \ldots$

$$
\mathscr{H}_{n+1}=\mathscr{H}_{(n+1)} \ominus \mathscr{H}_{(n)}
$$

and

$$
\mathscr{D}=\bigoplus_{k=0}^{\infty} \mathscr{H}_{k} \quad \text { (direct sum). }
$$

It follows that $\mathscr{D}$ is dense in

$$
\mathscr{H}=\bigoplus_{k=0}^{\infty} \mathscr{H}_{k} \quad \text { (Hilbert-sum) }
$$


and that

$$
\mathscr{H}_{k} \subseteq\left(\begin{array}{l}
\mathscr{H}_{k}^{1} \\
\mathscr{H}_{k}^{2}
\end{array}\right)
$$

As the $A^{i}$ are bounded Jacobi-fields we know that for all $\phi_{k}^{i} \in \mathcal{M}_{k}^{i}$

$$
A^{i}(x) \phi_{k}^{i}=\bigoplus_{j=k-1}^{k+1} A_{j k}^{i}(x) \phi_{k}^{i}
$$

holds with $A_{j k}^{i}(x) \in \mathcal{L}\left(\mathcal{H}_{k}^{i}, \mathscr{H}_{j}^{i}\right)$. Thus $A(x)$ as defined by (4.9) is in particular well defined on all vectors of the form

$$
\left(\begin{array}{l}
\phi_{k}^{1} \\
\phi_{k}^{2}
\end{array}\right), \quad \phi_{k}^{i} \in \mathscr{H}_{k}^{i} .
$$

Therefore if $Q_{k}$ denotes the orthogonal projection from

$$
\begin{gathered}
\left(\begin{array}{l}
\mathcal{H}_{k}^{1} \\
\mathcal{H}_{k}^{2}
\end{array}\right) \text { onto } \mathscr{H}_{k} \subseteq\left(\begin{array}{l}
\mathscr{H}_{k}^{1} \\
\mathcal{H}_{k}^{2}
\end{array}\right) \text { we can define for } x \in E \\
A_{j k}(x)=Q_{j} A(x) Q_{k} \in \mathcal{L}\left(\mathscr{H}_{k}, \mathscr{H}_{j}\right)
\end{gathered}
$$

and then for all $\phi=\left\{\phi_{0}, \cdots, \phi_{N}, 0,0, \cdots\right\} \in \mathscr{D}$ we get

$$
A(x) \phi=\sum_{k=0}^{N} \sum_{|j-k|<1} A_{j k}(x) \phi_{k} \quad \text { for all } x \in E .
$$

This proves that $A$ is bounded Jacobi-field in $\mathscr{H}$ with cyclic unit vector $\phi_{0}$ and domain $\mathscr{D}$. By (4.11) it follows $T \in \mathcal{E}_{J b}(\underline{E})$.

\section{IV.2. Topological stability.}

In order to study topological stability of $\mathscr{B}_{J}^{+}(\underline{\hat{E}})$ we exhibit a 'topology' (a notion of convergence) with respect to which $\mathscr{B}\left({ }_{J}^{+} \underline{\hat{E}}\right)$ is complete.

To this end suppose that we are given a net $\left(B^{\alpha}\right), \alpha \in A$, in $\mathscr{B}_{J}^{+}(\hat{E})$ which satisfies

i) $\left\{B^{\alpha}, \alpha \in A\right\}$ is pointwise bounded

ii) $\left(B^{\alpha}\right), \alpha \in A$, is pointwise Cauchysch

e.g. for all $\underline{\hat{a}}, \underline{\hat{b}} \in \underline{\hat{E}}$ we have

i) $\sup _{\infty \in A}\left|B^{\infty}(\underline{\hat{a}}, \underline{\hat{b}})\right|=C(\underline{\hat{a}}, \underline{\hat{b}})<\infty$

ii) $\left(B^{a}(\underline{\hat{a}}, \underline{\hat{b}})\right), \alpha \in A$, is a Cauchynet in $\mathbb{C}$.

By Cor. III.5.1.2 of [11] it follows that there is a continuous seminorm $q$ on $\underline{\underline{E}}$ such that

$$
\left|B^{\infty}(\underline{\hat{a}}, \underline{\hat{b}})\right| \leq q(\hat{a}) q(\hat{b}) \quad \text { for all } \alpha \in A .
$$

By assumption ii) for each pair $(\underline{\hat{a}}, \underline{\hat{b}})$ in $\underline{\hat{E}} \times \underline{\hat{E}}$ there is a complex number $B(\underline{\hat{a}}, \underline{\hat{b}})$ such that 


$$
B(\hat{a}, \underline{\hat{b}})=\lim _{\boldsymbol{\omega} \in \boldsymbol{A}} B^{\alpha}(\hat{a}, \underline{\hat{b}}) .
$$

It follows that $(\underline{\hat{a}}, \underline{\hat{b}}) \mapsto B(\underline{\hat{a}}, \underline{\hat{b}})$ defines a normalized sesquilinear form $B$ on $\underline{\hat{E}}$ which is positive and satisfies (4.19). Thus $B$ is continuous. Obviously the orthogonality relations ( 0$)$ and the symmetry relations (S) are stable with respect to pointwise limits, therefore $B \in \mathscr{B}_{0 s}^{+}(\underline{\hat{E}})$.

By nuclearity of $\underline{\hat{E}}$ its topology is defined by a family of Hilbert seminorms. Thus there are Hilbert seminorms $p$ and $h$ on $\underline{\hat{E}}$ such that

$$
q \leq p \leq h
$$

and such that the canonical map $\chi$ from $\mathscr{H}_{k}$ into $\mathscr{H}_{p}$ is nuclear where $\mathscr{H}_{h}$ and $\mathscr{H}_{p}$ are the Hilbert spaces canonically associated with $h$ and $p$, e.g. there are continuous linear maps $\psi: \underline{\hat{E}} \rightarrow \mathscr{H}_{p}, \phi: \underline{\hat{E}} \rightarrow \mathscr{H}_{h}$ with dense range such that

$$
p(\underline{\hat{a}})^{2}=\langle\psi(\underline{\hat{a}}), \psi(\hat{a})\rangle_{p}, \quad h(\underline{\hat{a}})^{2}=\langle\phi(\underline{\hat{a}}), \phi(\underline{\hat{a}})\rangle_{h}
$$

and

$$
\psi(\hat{a})=\chi \circ \phi(\hat{a})
$$

for all $\underline{\hat{a}} \in \hat{E} .\left(\langle\cdot, \cdot\rangle_{p},\langle\bullet, \bullet\rangle_{h}\right.$ : the scalar products of $\mathscr{H}_{p}$ respectively of $\left.\mathscr{H}_{h}\right)$. By (4.19), (4.21) and (4.22) it follows that

$$
(\psi(\hat{a}), \psi(\underline{\hat{b}})) \mapsto B^{\infty}(\hat{a}, \underline{\hat{b}})
$$

is a well defined quadratic form on $\psi(\hat{E}) \subset \mathscr{H}_{p}$ with

$$
\left|B^{\alpha}(\underline{\hat{a}}, \underline{\hat{b}})\right| \leq\|\psi(\hat{a})\| \cdot\|\psi(\hat{b})\| \text { for all } \alpha \in A .
$$

Thus it extends uniquely to a continuous nonnegative quadratic form on $\mathscr{H}_{p}$. Therefore there are nonnegative selfadjoint operators $C^{\alpha}$ in $\mathscr{H}_{p}$ with

$$
B^{\alpha}(\hat{a}, \hat{b})=\left\langle C^{\infty} \psi(\hat{a}), C^{\infty} \psi(\hat{b})\right\rangle \quad\left\|C^{\infty}\right\| \leq 1
$$

for all $\alpha \in A$ and all $\hat{a}, \underline{\hat{b}} \in \underline{\hat{E}}$. The same applies to $B$. There is a nonnegative selfadjoint operator $C$ in $\mathscr{H}_{p}$ with

$$
B(\hat{a}, \underline{\hat{b}})=\langle C \psi(\hat{a}), C \psi(\hat{b})\rangle \quad \text { for all } \underline{a}, \underline{\hat{b}} \in \underline{\hat{E}},
$$

and by (4.20) it follows

$$
C^{2}=\mathrm{w}-\lim _{\omega \in A} C^{\infty} C^{\infty}
$$

(weak limit in $\mathcal{L}\left(\mathscr{H}_{p}, \mathscr{H}_{p}\right)$ ).

As $\mathscr{H}_{p}$ is separable and as $\left\|C^{\alpha} C^{\infty}\right\| \leq 1$ for all $\alpha \in A$ there is a subsequence $\alpha(j)$, $j \in \mathbb{N}$ such that

$$
C^{2}=\underset{j \rightarrow \infty}{\mathrm{w}-\lim } C^{\alpha(j)} C^{\alpha(j)}
$$

Let us denote $C^{j}=C^{\infty(j)}, j \in \mathbb{N}$, and correspondingly $B^{j}=B^{\alpha(j)}$. The $B^{j}$ 
are supposed to satisfy the density condition (D). Thus if $\underline{\hat{a}} \in \underline{\hat{E}}$ and $\varepsilon>0$ are fixed there is (by (4.4)) $\underline{\hat{b}}_{j} \in \underline{E}_{0}$ such that

$$
B^{j}(\underline{\hat{a}}, \underline{\hat{a}})-\varepsilon \leq\left|B^{j}\left(\underline{\hat{a}}, \underline{\hat{b}}_{j}\right)\right|^{2} B^{j}\left(\hat{b}_{j}, \underline{\hat{b}}_{j}\right)^{-1} \quad \text { for all } j \in \mathbb{N}
$$

e.g.

$$
\left\|C^{j} \psi(\hat{a})\right\|^{2}-\varepsilon \leq\left|\left\langle C^{j} \psi(\underline{\hat{a}}), C^{j} \psi\left(\hat{b}_{j}\right)\right\rangle\right|^{2}\left\|C^{j} \psi\left(\underline{b}_{j}\right)\right\|^{-2} \text { for all } j \in \mathbb{N} .
$$

As the right hand side of this inequality is scale invariant with respect to $\hat{b}_{j}$ we can and will assume that the $\underline{\hat{b}}_{j}$ are normalized according to

$$
\left\|\phi\left(\hat{b}_{j}\right)\right\|_{\mathscr{A}_{h}}=1
$$

where $\phi$ is given by (4.22) (Note that by definition and (4.22) only those $\hat{b}_{j}$ occur in (4.27) for which $\phi\left(\hat{b}_{j}\right) \neq 0$ as we may assume that the lefthand side of (4.27) is positive).

Therefore by weak compactness of the closed unitball of $\mathscr{H}_{h}$ we can find a subsequence $i(i), i \in \mathbb{N}$, and $\phi_{0} \in \mathscr{H}_{k}$ such that

$$
\phi_{0}=\mathrm{w}-\lim _{j \rightarrow \infty} \phi\left(\hat{b}_{j(i)}\right)
$$

in $\mathscr{H}_{h}$. By (4.22) and nuclearity of $\chi$ it follows

$$
\psi_{0}=\chi\left(\phi_{0}\right)=\lim _{i \rightarrow \infty} \chi \circ \phi\left(\hat{b}_{j(i)}\right)=\lim _{i \rightarrow \infty} \psi\left(\hat{b}_{j(i)}\right)
$$

in $\mathscr{H}_{p}$ and

$$
\psi_{0} \in\left[\psi\left(\underline{\hat{E}}_{0}\right)\right] \subseteq \mathscr{H}_{p} .
$$

In order to prove

$$
\lim _{i \rightarrow \infty}\left\|C^{j(i)} \psi\left(\hat{b}_{j(i)}\right)\right\|^{2}=\left\|C \psi_{0}\right\|^{2}
$$

we write $\psi\left(\hat{b}_{j(i)}\right)=\psi_{0}+\phi_{i}$ with $\phi_{i} \underset{i \rightarrow 0}{\rightarrow} 0$ in $\mathscr{H}_{p}$ and estimate

$$
\begin{aligned}
& \|\| C^{j(i)} \psi\left(\hat{b}_{j(i)}\right)\left\|^{2}-\right\| C \psi_{0}\left\|^{2}\left|\leq\|\| C^{j(i)} \psi_{0}\left\|^{2}-\right\| C \psi_{0} \|^{2}\right|+\right. \\
& \quad+\left\|C^{j(i)}\right\|^{2} \cdot\left(\left\|\phi_{i}\right\|^{2}+2\left\|\psi_{0}\right\|\left\|\phi_{i}\right\|\right) .
\end{aligned}
$$

Thus (4.26) implies (4.32). And similarly (4.26) and (4.30) imply

$$
\lim _{i \rightarrow \infty}\left\langle C^{j(i)} \psi(\hat{a}), C^{j(i)} \psi\left(\hat{b}_{j(i)}\right)\right\rangle=\lim _{i \rightarrow \infty}\left\langle\left(C^{j(i)}\right)^{2} \psi(\hat{a}), \psi\left(\hat{b}_{j(i)}\right)\right\rangle=\left\langle C^{2} \psi(\hat{a}), \psi_{0}\right\rangle .
$$

Therefore taking the $\lim i \rightarrow \infty$ of (4.27) yields

$$
\|C \psi(\underline{a})\|^{2}-\varepsilon \leq\left|\left\langle C \psi(\hat{a}), C \psi_{0}\right\rangle\right|^{2} \cdot\left\|C \psi_{0}\right\|^{-2} .
$$

By relation (4.24) the sesquilinear form $B$ is realized in the Hilbert space $[C \psi(\underline{\hat{E}})] \subset \mathscr{H}_{p}$.

Relation (4.31) and inequality (4.33) imply that $C \psi\left(\hat{E}_{0}\right)$ is dense in this Hilbert space $[C \psi(\underline{\hat{E}})]$. This shows finally that the weak limit $B$, too, satisfies 
the density condition (D) and thus proves

Theorem 4.5. Any net $\left(B^{\infty}\right), \alpha \in A$, in $\mathscr{B}_{J}^{+}(\underline{\hat{E}})$ which is Cauchysch in the sense of (4.18) has a limit $B$ in $\mathscr{B}_{J}^{+}(\hat{E})$.

Corollary 4.6. Any net of Jacobi-states $\left(T^{\infty}\right), \alpha \in A$, in $\mathcal{E}_{J}(\underline{E})$ such that the associated net $\left(B^{\infty}\right), \alpha \in A,\left(\right.$ by Th. 3.7) in $\mathscr{B}_{J}^{+}(\hat{E})$ satisfies (4.18) has a Jacobistate $T$ as limit.

\section{§V. Conclusions}

If a space $E$ satisfies $(\mathrm{H})$ and has property (a) then by the preceding investigations we know many details about states on the (complete) tensor algebra $\underline{E}$ over $E$. And the information about these states goes considerably beyond that provided by the GNS-construction.

1. Various classes of states on $\underline{E}$ have been distinguished. Some of them have been characterized. Among these the set of Jacobi-states appears in a natural way as the set of those states on the tensor algebra $\underline{E}$ which reflect the graded structure of this algebra in a direct way. A certain subset of the components of the Jacobi-decomposition for these states provides the "parametrization" of these states as indicated in section O. (Lemma 3.5 and Proposition 3.7). This can be viewed as the beginning of a complete classification of the set of states on $\underline{E}$.

2. There are "very many" Jacobi-states. Already the weak sequential closure of the set of bounded Jacobi-states $\mathcal{E}_{J b}(\underline{E})$ equals the set of all states on $\underline{E}$ (Corollary 2.4), and $\varepsilon_{J b}(\underline{E})$ is convex (Proposition 4.4). (In a finite dimensional situation these two properties together would mean that only boundary-points may not belong to the set in question).

3. In terms of the Jacobi-decomposition a "topology" on the set $\varepsilon_{J}(\underline{E})$ of Jacobi-states has been given with respect to which $\mathcal{E}_{J}(\underline{E})$ is complete (Corollary 4.6). This topology $\sigma_{0}$ is a "natural" topology on the set of all Jacobidecompositions but not on the set of states on $\underline{E}$. It is considerably finer than the weak topology $\sigma=\sigma\left(\underline{E}^{\prime}, \underline{E}\right)$ on the set of states on $\underline{E}$. Though a one-toone correspondence between Jacobi-states and Jacobi-decompositions could be established (Theorem 3.7) it is still an open problem whether $\varepsilon_{J}(E)$ is also complete with respect to $\sigma$ (if so Corollary 2.4 would imply that the set of Jacobi-states equals the set of states on $\underline{E}$ ). Theorem 4.5 indicates that this might not be the case in contrast to the claim in our first version. In any case as indicated in 2, there are not "too many" states on $\underline{E}$ which are not 
Jacobi-states.

4. In general relativistic quantum field theory [2-6] the basic space $E$ is $E$ $=S\left(\mathbb{R}^{4}\right) \otimes V$ with some finite dimensional vector space $V$ and $S\left(\mathbb{R}^{4}\right)$ the Schwartz space of rapidly decreasing $C^{\infty}$-functions on space-time. It is known that this space satisfies $(\mathrm{H})$ and according to Proposition $\mathrm{A}$ this space also has property (as). Thus our results apply and explain in rather general terms why every relativistic quantum field which has been constructed up to now on physical space-time is a Jacobi-field (not necessarily bounded) in accordance with our physical intuition about quantum fields. (If it would turn out that $\mathcal{E}_{J}(E)$ actually does not equal the set of all states on $\underline{E}$ then as indicated in 3 the states on $\underline{E}$ being not Jacobi-states could in some sense be considered as being exceptional).

5. If the functional characterization of Jacobi-states (Theorem 3.7) is applied in general relativistic quantum field theory it adds a lot of new information about the general structure of the "vacuum expectation values" of such a theory and gives new aspects of various problems (association of particle and fields, scattering theory, asymptotic completeness, combining locality and positivity, details about the energy momentum spectrum of the theory, construction of relativistic quantum field models).

6. It should be noticed that the positivity condition (P) for the Jacobidecomposition $B$ of a Jacobi-state $T$ on $\underline{E}$ provides in connection with Lemma 3.5 and Theorem 3.7 many "correlation-type inequalities" for the components $T_{n}$ of $T$. In order to illustrate this point we present a simple example. The components $B_{I, J}$ of $B$ can be expressed in terms of the given $n$-point-correlation functionals" $T_{n}$ (Th. 3.7 and Lemma 3.5). The positivity condition (P) implies in its simplest form

$$
\left|B_{I, J}(a, b)\right|^{2} \leq B_{I, I}(a, a) \cdot B_{J, J}(b, b) .
$$

This inequality says for instance (assuming $T_{1}=0$ for convenience and choosing $I, J$ appropriately):

$$
\left|\left(T_{5}-T_{2} \otimes T_{3}-T_{1} \otimes T_{4}\right)\right|^{2}\left(x_{2}^{*} \otimes x_{3}\right) \leq\left(T_{4}-T_{2} \otimes T_{2}-T_{3}(1) T_{3}\right)\left(x_{2}^{*} \otimes, x_{2}\right) F_{6}\left(x_{3}^{*} \otimes x_{3}\right)
$$

with $F_{6} \in E_{6}^{\prime}$ such that

$$
0 \leq F_{6}\left(x_{3}^{*} \otimes x_{3}\right) \leq\left(T_{6}-T_{3} \otimes T_{3}-\sum_{I, J \in \mathscr{g}_{1}(3)} B_{I, J}\right)\left(x_{3}^{*} \otimes x_{3}\right)
$$

for all $x_{3} \in E_{3}$. Here (1) denotes the "1-convolution" according to Eq. (3.37) and $\sum_{I, J \in \mathscr{g}_{1}(3)} B_{I, J}$ is a function of $\left\{T_{2}, T_{3}, T_{4}\right\}$. 
7. The concept of a Jacobi-field has also proved to be very important in the problem of positive continuous linear extensions from finite sections

$$
\underline{E}_{(N)}=\left\{\underline{x} \in \underline{E} \mid x_{n}=0 \text { for all } n>N\right\}, N=0,1,2, \cdots
$$

to the whole tensoralgebra $\underline{E}$. This problem asks for necessary and sufficient conditions on a given functional $T_{(N)}=\left\{1, T_{1}, \cdots, T_{N}\right\} \in \underline{E}_{(N)}^{\prime}$ for the existence and uniqueness of a state $T$ on $\underline{E}$ such that its restriction $T \uparrow \underline{E}_{(N)}$ to the finite section $\underline{E}_{(N)}$ equals $T_{(N)}$. A nearly complete answer is given in reference [10]. 8. Finally we would like to point out two other applications of this analysis of states. The first applies the concept of a Jacobi-field to relativistic quantum field theory and uses it together with a solution of the above extension problem to enlarge the class of known models of relativistic quantum field theory on physical space time considerably [17]. The second application relies on an extension of this analysis to a stronger positivity condition. It provides a method of constructing Radon-probability measures on the dual $E^{\prime}$ of the basic space $E$ in terms of given moments. In particular a way of constructing such measures which are not Gaussian measures (or slight modifications there of) is obtained [18]. This then illustrates point iii) of the introduction.

Appendix: On spaces with approximation property (a).

Properties (a) and (as) of Definition 2.1 are related to but are in general not identical with the approximation property in the general theory of topological vector spaces (for instance $\S 13$ of [13]). The approximation property (in general) means that there is a net $\left(\pi_{\gamma}\right)_{\gamma \in \Gamma} \subset \mathcal{L}(E, E)$ such that

i) $\operatorname{dim} \operatorname{Ran} \pi_{\boldsymbol{\gamma}}<\infty$

ii) $\lim _{\gamma \in \Gamma} \pi_{\gamma}(x)=x$ uniformly on precompact subsets of $E$.

But in general it is not known whether this net also is pointwise bounded. This property however is crucial for our reasoning. Therefore we had to use property (a).

Obviously for all locally convex topological vectorspaces $E$ for which the pointwise convergence of a pointwise bounded net of continuous linear maps $E \rightarrow E$ implies its uniform convergence on all precompact subsets of $E$ property (a) implies the general approximation property. By the BanachSteinhaus Theorem this is the case for all barreled spaces. Moreover, whenever a barreled space $E$ has a weak Schauder basis ( $\$ 43.5$ of [13]) $E$ has the general approximation property and by the proof of this fact ( $\$ 43.5 .3$ of [13]) also property (a) with respect to sequences. 
Instead of trying to give a systematic treatment of property (a) we discuss some simple facts about it and are thus able to show that many of those spaces which are actually used in applications indeed have property (a) respectively (as).

A first immediate observation is:

(1) If a locally convex topological vector space $E$ is topologically isomorphic to a locally convex topological vector $F$ which has property (a) respectively (as) then $E$ has property (a) respectively (as).

The proof of the following three hereditary properties is similar but simpler than the proof of the corresponding statements about the general approximation property ( $\$ 43.4$ of [13]) and is therefore omitted:

(2) If a locally convex space $E$ has property (a) respectively (as) then every complemented subspace of $E$ has property (a) respectively (as).

(3) The locally convex direct sum $E=\bigoplus_{\omega} E_{\alpha}$ of locally convex spaces $E_{\alpha}$ has property (a) iff all $E_{\alpha}$ have property (a).

(4) The topological product $E=\prod E_{\alpha}$ of locally convex spaces $E_{\alpha}$ has property (a) iff all $E_{\alpha}$ have property (a).

It is fairly obvious that the nuclear Fréchet space $s$ of rapidly decreasing sequences has property (as). By a theorem of T. Kömura and Y. Komura (p. 212 of [15] and [14]) it is known that every nuclear space is topologically isomorphic to a subspace of the product space $s^{\wedge}$ for some index set $\wedge$ (every metrizable nuclear space is topologically isomorphic to a subspace of $\mathrm{s}^{\boldsymbol{N}}$ ). Therefore whenever a nuclear space is topologically isomorphic to a subspace of $\mathrm{s}^{\wedge}$ which is left invariant by the canonical projections of $\mathrm{s}^{\wedge}$ (this is used in the proof of (4)) it has property (a).

A class of locally convex spaces which is of particular interest in applications is the class of nuclear Fréchet spaces. We are going to show that these spaces have property (as). By properties (1)-(4) the class of spaces with property (a) is enlarged considerably.

Proposition A. Every nuclear Fréchet space E has property (as).

Proof. 1. By Corollary 7.3.3 of [11] we know that every nuclear Fréchet space $E$ is the projective limit of a sequence of separable Hilbert spaces $H_{n}$ (corresponding to Hilbertian seminorms $h_{n}$ on $E$ ) such that the spectral maps 
$L_{n, n+1}: H_{n+1} \rightarrow H_{n}, n=0,1,2, \cdots$, are nuclear.

According to Satz 4.2 of [12] each $H_{n}$ has an orthonormal basis

$$
\left\{\hat{e}_{j}^{n}=Q_{n}\left(e_{j}^{n}\right): j \in N\right\} \text { with }\left\{e_{j}^{n}: j \in N, n=0,1,2, \cdots\right\} \subset E
$$

and $Q_{n}: E \rightarrow H_{n}$ the canonical projections.

The spectral maps and the projections are related by

$$
Q_{p}=L_{p, q} Q_{q}=L_{p, p+1} \cdots L_{q-1, q} Q_{q} \quad \text { for } q>p .
$$

Nuclearity of $L_{n, n+1}$ implies furthermore that we can choose these basis $\left\{\hat{e}_{j}^{n} \mid\right.$ $j \in N\}, n=0,1,2, \cdots$, such that for all $x \in H_{n+1}$

$$
L_{n, n+1}(x)=\sum_{j=1}^{\infty} \lambda_{j}^{n+1}\left\langle\hat{e}_{j}^{n+1}, x\right\rangle_{n+1} \hat{e}_{j}^{n} \quad \text { with } \sum_{j=1}^{\infty}\left|\lambda_{j}^{n+1}\right|<\infty .
$$

2. Now by (A.2) we have

$$
L_{n, n+1}\left(\hat{e}_{j}^{n+1}\right)=\lambda_{j}^{n+1} \hat{e}_{j}^{n}
$$

and therefore by (A.1)

$$
Q_{n}\left(e_{j}^{n+1}-\lambda_{j}^{n+1} e_{j}^{n}\right)=0 .
$$

This shows that moreover the $e_{j}^{n}$ can be choosen in such a way that

$$
e_{j}^{n+1}=\lambda_{j}^{n+1} e_{j}^{n} \quad n, j \in \mathbb{N}
$$

holds. Iterating this equation we get

$$
e_{j}^{n}=\lambda_{j}^{(n)} e_{j}^{0} \quad \text { where } \quad \lambda_{j}^{(n)}=\lambda_{j}^{n} \cdots \lambda_{j}^{2} \lambda_{j}^{1} .
$$

For any $x \in E$ and $p \in N$ we express $Q_{0}(x)$ and $Q_{p}(x)$ in terms of the basis of $H_{0}$ respectively of $H_{p}$ :

$$
Q_{0}(x)=\sum_{j=1}^{\infty}\left\langle\hat{e}_{j}^{0}, Q_{0}(x)\right\rangle_{0} \hat{e}_{j}^{0} \quad Q_{p}(x)=\sum_{j=1}^{\infty}\left\langle\hat{e}_{j}^{p}, Q_{p}(x)\right\rangle_{p} \hat{e}_{j}^{p} .
$$

Because of (A.1) and (A.5) we get

$$
Q_{0}(x)=L_{0, p}\left(Q_{p}(x)\right)=\sum_{j=1}^{\infty}\left\langle\hat{e}_{j}^{p}, Q_{p}(x)\right\rangle_{p} L_{0, p}\left(\hat{e}_{j}^{b}\right)=\sum_{j=1}^{\infty}\left\langle\hat{e}_{j}^{b}, Q_{p}(x)\right\rangle_{p} \lambda_{j}^{(p)} \hat{e}_{j}^{0}
$$

and thus by (A.6) for all $j, p \in N$ and all $x \in E$

$$
\left\langle\hat{e}_{j}^{0}, Q_{0}(x)\right\rangle_{0}=\lambda_{j}^{(p)}\left\langle\hat{e}_{j}^{p}, Q_{p}(x)\right\rangle_{p} .
$$

3. Now the construction of continuous linear maps $\pi_{N}: E \rightarrow E$ as required by property (as) is quite easy. For $x \in E$ define

$$
\pi_{N}(x)=\sum_{j=1}^{N}\left\langle Q_{0}\left(e_{j}^{0}\right), Q_{0}(x)\right\rangle_{0} e_{j}^{0} .
$$


Obviously $\pi_{N} \in \mathcal{L}(E, E)$ and $\operatorname{dim} \operatorname{Ran} \pi_{N}=N$.

Then define for $p=1,2 \ldots$

$$
\pi_{N}^{p}(x)=\sum_{j=1}^{N}\left\langle Q_{p}\left(e_{j}^{p}\right), Q_{p}(x)\right\rangle_{p} e_{j}^{p} .
$$

Equations (A.5) and (A.7) imply

$$
\pi_{N}^{p}(x)=\pi_{N}(x) \quad \text { for all } x \in E, p, N \in \mathbb{N}
$$

and therefore for arbitrary but fixed $p=0,1,2, \cdots$

$$
h_{p}\left(x-\pi_{N}(x)\right)=h_{p}\left(x-\pi_{N}^{p}(x)\right)=\left\|Q_{p}(x)-Q_{p}\left(\pi_{N}^{p}(x)\right)\right\|_{p} \underset{n \rightarrow \infty}{\rightarrow} 0,
$$

if we observe (A.6) and

$$
Q_{p}\left(\pi_{N}^{p}(x)\right)=\sum_{j=1}^{N}\left\langle e_{j}^{p}, Q_{p}(x)\right\rangle_{p} e_{j}^{p} .
$$

This proves

$$
\lim _{N \rightarrow \infty} \pi_{N}(x)=x \quad \text { in } E
$$

for every $x \in E$, that is this space $E$ has property (as).

\section{Acknowledgments}

The main part of the work for this article was done in the stimulating atmosphere of the RIMS, Kyoto University, as a JSPS-fellow. I would like to take this opportunity to express my sincere thanks to the Japan Society for the Promotion of Science for this fellowship. It is a great pleasure to thank Prof. H. Araki not only for several discussions on this subject but also for his attentive hospitality. This article was eventually finished at the CPT, CNRS Luminy, Marseille. I want to thank the CPT, in particular M. SirugueCollin and M. Sirugue, for the kind hospitality I enjoy there. Last but not least, I express my sincere thanks to Prof. H.J. Borchers and J. Yngvason; to the latter for pointing out two not unimportant gaps in the first version and to the former for several encouraging discussions at various stages in the preparation of this article.

\section{References}

[1] Dieudonné, J., Treatise cn Analysis, 10-11, Academic Press, New York and London 1970, p. $406 \mathrm{ff}$.

[2] Borchers, H.J., On the structure of the algebra of field operators, Nuov. Cimento 24, (1962), 214-236. 
[3] Powers, R.T., Self adjoint algebras of unbounded operators, Comm. Mcth. Phys. 21, (1971), 85-124.

[4] Maurin, K., General Eigenfunction Expansions and Unitary Representations of Topological Groups, PWN-Polish Scientific Publishers, Warszawa 1968.

[5] Bogolubov, N.N., Logunov, A.A., Todorov, I.I., Introduction to axiomatic field theory, London, Benjamin 1975 and references there.

[6] Borchers, H.J., Algebraic Aspect of Wightman field theory, in: Statistical Mechanics and Field Theory; Ed. R.N. Sen, C. Weil, Israel University Press, Jerusalem 1972.

[7] Brüning, E., On Jacobi-Fields; Colloquia Mathematica Societatis Janos Bolyai; 27. Random Fields; Esztergom (Hungary). 1979.

[8] - On the characterization of relativistic quantum field theories in terms of finitely many vacuum expectation values, Comm. Math. Phys. 58 (1978), I.: 139-160, and II: $167-194$.

[9] — The $n$-field-irreducible part of an $n$-point functional, Ann. Inst. Henri Poincaré Sect. A, ol, XXXIV n. 3, (1981), 309-328.

[10] - Monotone continuous linear extensions on tensor-algebras, RIMS preprint, December 1983.

[11] Schaefer, H.H., Topological Vector Spaces, Springer Verlag, New-York, Heidelberg, Berlin, 1970.

[12] Floret, K., Wloka, J., Einführung in die Theorie der lokalkonvexen Raüme; Lecture Notes in Mathematics 56, Springer Verlag Berlin Heidelberg, New York 1968.

[13] Köthe, G., Topological Vector Spaces II. Springer Verlag, New York Heidelberg Berlin 1979.

[14] Kōmura, T., Komura. Y., Über die Einbettung der nuklearen Räume in (S), Math. Ann. 162, (1966), 284-286.

[15] Wong, Y.C., Schwartz spaces, Nuclear Spaces and Tensor Products, Lecture Notes in Mathematics 726; Springer Verlag, Berlin, Heidelberg, New York 1979.

[16] Alcantara, J. Dubin, D.A, I*-algebras and their applications, Publ. RIMS, Kyoto Univ. 17, (1981), 179-199.

[17] Brüning, E., A new class of Wick powers of generalized frce fields, J. Math. Phys. 25 (1984), 3064.

[18] - On the construction of Radon-Probability measures on infinite dimensional spaces I, preprint $N$. 110 of project $n$. 2, mathematics + phystcs, ZiF, University of Bielefeld, Aug. 1984.

[19] Berezanskii, Yu. M. Koshmanenko, Axiomatic field theory in terms of operator Jacobian matrices, Dokl. Akod. Nauk. SSSR, 1969.

[20] Ruelle, D., Statistical Mechanics, chapter 4, W.A. Benjamin, Inc., New York, 1969.

[21] Dobrushin, R.I., Minlos, R.A., a) The Moments and Polynomials of a Generalized Random Field; Theory of Probability and its Applications; XXIII, 1978, No. 4, 686-699.

b) Polynomials in Linear Random Functions; Russian Math. Survey 32; 2 (1981); from Uspekhi Mat. Nauk. 32: 2 (1977), 67-122. 